Extended smoothed boundary method for solving partial differential equations with general boundary conditions on complex boundaries

This article has been downloaded from IOPscience. Please scroll down to see the full text article.

2012 Modelling Simul. Mater. Sci. Eng. 20075008

(http://iopscience.iop.org/0965-0393/20/7/075008)

View the table of contents for this issue, or go to the journal homepage for more

Download details:

IP Address: 141.211.173.82

The article was downloaded on 25/06/2013 at 20:33

Please note that terms and conditions apply. 


\title{
Extended smoothed boundary method for solving partial differential equations with general boundary conditions on complex boundaries
}

\author{
Hui-Chia Yu, Hsun-Yi Chen and K Thornton \\ Department of Materials Science and Engineering, University of Michigan, Ann Arbor, MI, USA \\ E-mail: kthorn@umich.edu
}

Received 17 January 2012, in final form 24 July 2012

Published 14 September 2012

Online at stacks.iop.org/MSMSE/20/075008

\begin{abstract}
In this paper, we describe an approach for solving partial differential equations with general boundary conditions imposed on arbitrarily shaped boundaries. A continuous function, the domain parameter, is used to modify the original differential equations such that the equations are solved in the region where a domain parameter takes a specified value while boundary conditions are imposed on the region where the value of the domain parameter varies smoothly across a short distance. The mathematical derivations are straightforward and applicable to a wide variety of partial differential equations. To demonstrate the general applicability of the approach, we provide four examples herein: (1) the diffusion equation with both Neumann and Dirichlet boundary conditions; (2) the diffusion equation with both surface diffusion and reaction; (3) the mechanical equilibrium equation; and (4) the equation for phase transformation with the presence of additional boundaries. The solutions for several of these cases are validated against numerical solutions of the corresponding sharpinterface equations. The potential of the approach is demonstrated with five applications: surface-reaction-diffusion kinetics with a complex geometry, Kirkendall-effect-induced deformation, thermal stress in a complex geometry, phase transformations affected by substrate surfaces and relaxation of a droplet on irregular surfaces.
\end{abstract}

(Some figures may appear in colour only in the online journal)

\section{Introduction}

The smoothed boundary method (SBM) [1-3] has recently been demonstrated as a powerful tool for solving diffusion equations with no-flux boundary conditions imposed at irregular boundaries within the computational domain. The method's origin can be traced to the embedded boundary method and the immersed interface (boundary) method, both of which embed a more complicated domain in a computational box with simpler geometry. These 
methods eliminate the need for a structural mesh when solving partial differential equations within the embedded geometries because the grid system is obtained by a discretization of the regular computational box. (For an overview, see [4-10].) To impose boundary conditions at the immersed interfaces, a discretized Dirac delta function is employed to distribute a singular source over nearby grid points. Various studies have examined optimal discretization of the Dirac delta function [7, 8, 11, 12]. Similarly, the level set method can also be considered an immersed-interface-type method because the boundary defined by the contour of the zero level set is embedded within a regular computational box. Although the level set method was developed mainly for tracking moving boundaries [13], it is also applicable for solving partial differential equations with boundary conditions imposed at the zero-level-set contour using a technique similar to the immersed interface method [14,15]. In addition to the methods above, the phase-field approach possesses certain similarities to embedding interfaces within the computational box and also has the significant advantage of avoiding the need to explicitly track the interfaces. However, phase-field methods are not widely employed in simulations that involve explicit boundary conditions along interfaces. While the GibbsThomson boundary condition is automatically imposed in the standard phase-field model, there have been only a few studies in which phase-field models were used with explicit boundary conditions at interfaces. For example, in solidification problems, equilibrium conditions, such as equilibrium temperature or concentration [16,17], are imposed at solid-liquid interfaces in which the order parameter field and the temperature field are coupled via a latent heat term. Except for this type of phase-field model, the direct application of boundary conditions at interfaces is rarely used because the construction of boundary conditions requires the tedious process of formally including an additional energy term in the energy functional, as suggested by Cahn [18]. Examples of imposed boundary conditions in the phase-field model using modified energy functionals can be found in the recent works of Warren et al [19,20] and Qian et al [21].

In contrast to the techniques for distributing a singular source of boundary conditions to grid points near the interfaces in the immersed interface method, the SBM spreads the zero-thickness boundary into a finite-thickness diffuse interface using a phase-fieldlike, continuously transitioning domain indicator function (hereinafter termed the domain parameter'). Mathematically, this method approximates a Heaviside step function as a hyperbolic tangent function having one specified uniform value in a domain and continuously changing its value across the interface to another value specifying the other domain. Therefore, boundary conditions are straightforwardly distributed among the grid points residing within the interfacial regions in which the domain parameter varies smoothly across a short distance. This method has been successfully employed in simulating diffusion processes $[22,23]$ and wave propagation [1-3, 24, 25] constrained within geometries described by a domain parameter with a no-flux boundary condition imposed on the diffuse interfaces. Similar approaches have also been proposed to solve differential equations constrained in domains defined by order parameters in the phase-field model [26-28]. These works demonstrated the potential for this type of numerical method that circumvents the difficulties associated with constructing a finite element mesh (e.g. meshing the surface and then building a volumetric mesh based on the surface mesh or combining regular subdomains that can be easily meshed). Such an approach is particularly useful when complex structures are involved. However, the method was only applicable to no-flux boundary conditions, and no further extensions to other types of equations or boundary conditions have been reported. Recently, Lowengrub and coworkers [29-35] developed an alternative formulation for solving partial differential equations with various boundary conditions, based on asymptotic analyses commonly conducted in phasefield modeling, which is different from the general derivation of the SBM presented in this 
paper. Although such an implementation for imposing boundary conditions differs from the 'formal' practice suggested by Cahn [18], it dramatically simplifies the formulation, provides a justification of the method, and increases the applicability of the approach.

In this study, we provide a mathematically consistent SBM and a precise derivation for the equations, such that the method is generalized from its limited original application to a wide range of differential equations and boundary conditions. We consider the following specific equations: (1) the diffusion equation with Neumann and/or Dirichlet boundary conditions; (2) the bulk diffusion equation coupled with surface diffusion and reaction; (3) the mechanical equilibrium equation for linear elasticity; and (4) the Allen-Cahn or Cahn-Hilliard equations with contact angles as boundary conditions. Note that although the original SBM [1-3] used a spectrum method to solve the resulting differential equations, we chose the finite difference method to solve our SBM formulated equations in real space in this paper to demonstrate the versatility of the method. Some of the results of the reformulated equations are compared with those of their corresponding original equations. The errors are provided and their general behavior is studied. In this paper, we emphasize the convenience and versatility of the formulation, instead of the in-depth investigations for each individual problem presented or the purely mathematical perspective of the error resulting from the reformulation. The method is especially useful for three-dimensional image-based simulations because of its efficiency and flexibility in handling complex geometries without structural-mesh techniques.

\section{Background}

The method is based on a diffuse-interface description of different phases, similar to the continuously transitioning order parameters in the phase-field method [36-41] often employed in simulating phase transformations and microstructural evolutions in materials. Phase field models are based on thermodynamics and kinetics of a multiphase system, in which phases (e.g. liquid, solid, vapor, or two solids or liquids with different compositions) are described by one or more order parameters with prescribed bulk values for each phase. At the interface, the order parameter changes in a controlled manner. Asymptotic analyses [41] can be used to show that the phase-field governing equations approach the corresponding sharp-interface equations in the sharp-interface limit.

Despite the advantages of phase-field-type diffuse-interface methods for front tracking problems, we focus on another important advantage for efficiently solving differential equations within diffuse-interface-defined domains. Here, we adopt the concept to describe internal domain boundaries with an order-parameter-like domain parameter, which may or may not be stationary and takes a value of 1 inside the domain of interest and 0 outside. The equations are solved where the domain parameter is 1 , with boundary conditions imposed where the domain parameter is at an intermediate value (approximately 0.5). Figure 1 schematically illustrates the sharp and diffuse interfaces. In the conventional sharp-interface description, the domain of interest is $\Omega$ and is bounded by a zero-thickness boundary, denoted $\partial \Omega$; see figure $1(a)$. Within $\Omega$, the partial differential equations are solved according to the boundary conditions imposed at $\partial \Omega$. Conversely, in the diffuse-interface description, we employ a continuous domain parameter, which is uniformly 1 within the domain of interest and uniformly 0 outside. In this case, the originally sharp domain boundary is smoothed to yield a diffuse interface with a finite thickness given by $0<\psi<1$; see figure $1(b)$. The system thus determines the boundary by variation of the domain parameter. In addition, the gradient of the domain parameter $\nabla \psi$ automatically determines the inward normal vector of the contour level sets of $\psi$; see figure $1(c)$. Our goal is to solve partial differential equations within the region where $\psi=1$ while imposing boundary conditions at the narrow transitioning interfacial 

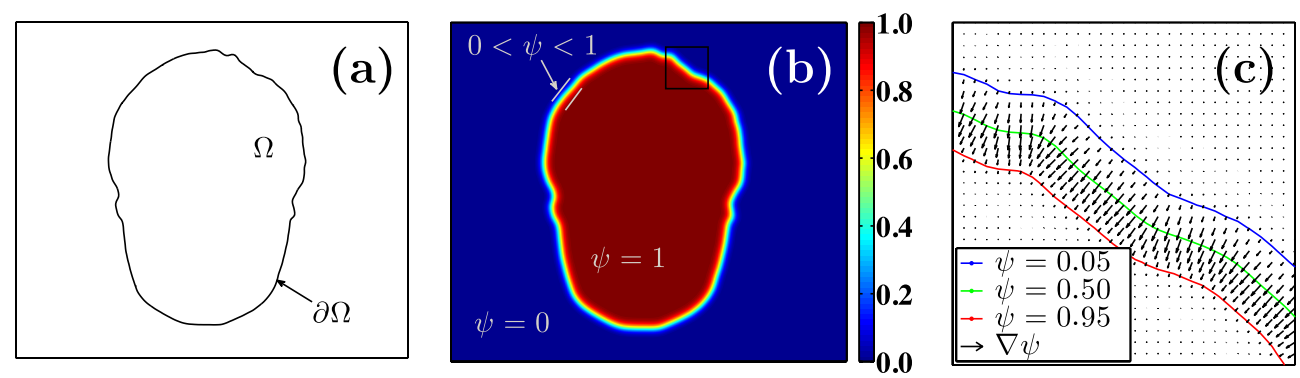

Figure 1. (a) Conventional sharp-interface description of a domain bound by a zero-thickness boundary. (b) Diffuse interface domain and boundary defined by a continuous domain parameter, $\psi$. (c) Inward normal vectors defined by $\nabla \psi$ plotted for the square region in $(b)$.

region where $0<\psi<1$. However, the convention can be reversed such that the domain is defined by $\psi=0$, in which case the following derivation could be modified by replacing $\psi$ with $1-\psi$ accordingly. This could be used to solve a problem where multiple equations govern different regions within the computational domain. Furthermore, these equations can be coupled through the shared boundary conditions, making the method highly versatile.

\section{Formulation}

\subsection{General approach}

The general approach is as follows. The domain parameter describes the domain of interest ( $\psi=1$ inside the domain and $\psi=0$ outside). The transition between the two values described must be smooth so that the gradient is well defined. In this work, we have assumed the domain parameter to take the form of a hyperbolic tangent function for three reasons. First, it can be numerically implemented with ease. Second, it is consistent with the solution to the phase-field equations, and thus this choice allows coupling of the two approaches; that is, one could for example simulate microstructural evolution using the phase-field model, but impose interfacial flux boundary condition using the SBM. Third, when given a non-smooth microstructural data, one could use the phase-field equations or level-set distancing method to obtain the domain parameter from the discontinuous data. Other forms of domain parameters are possible, as long as the transition is monotonic and the gradient of the domain parameter has a narrow peak in the interfacial region. A better convergence could possibly be obtained using a function that has a more confined gradient; however, examining other forms of the domain parameter is beyond the scope of this paper.

As an example, we consider the Laplacian of the function, $H$. As the first step in deriving formulation for the Neumann boundary condition, $\nabla^{2} H$ is multiplied by the domain parameter, $\psi$. Using identities of the product rule of differentiation such as

$$
\psi \nabla^{2} H=\nabla \cdot(\psi \nabla H)-\nabla \psi \cdot \nabla H,
$$

we obtain terms proportional to $\nabla \psi$. Because the inward unit normal of the boundary (pointing to the regions where $\psi=1), \vec{n}$, is given by $\nabla \psi /|\nabla \psi|$, such terms can be written in terms of $\partial H / \partial n=\nabla H \cdot \vec{n}=\nabla H \cdot \nabla \psi /|\nabla \psi|$, and thus reformulated to be the Neumann boundary condition imposed on the diffuse interface.

Similarly, to derive the SBM formulation for the Dirichlet boundary condition, the differential equation is multiplied by the square of the domain parameter. Again using 
mathematical identities, $\psi^{2} \nabla^{2} H=\psi \nabla \cdot(\psi \nabla H)-\psi \nabla \psi \cdot \nabla H$, where $\psi \nabla \psi \cdot \nabla H=$ $\nabla \psi \cdot \nabla(\psi H)-H|\nabla \psi|^{2}$, we obtain

$$
\psi^{2} \nabla^{2} H=\psi \nabla \cdot(\psi \nabla H)-\left[\nabla \psi \cdot \nabla(\psi H)-H|\nabla \psi|^{2}\right] .
$$

Note that $H$, associated with $|\nabla \psi|^{2}$ appearing in the last term, is the boundary value, $\left.H\right|_{\partial \Omega}$, imposed on the diffuse interface. Specific details of the derivation depend on the equation to which the approach is applied, and we therefore provide four examples below.

\subsection{Diffusion equation}

The first example is the diffusion equation with Neumann and/or Dirichlet boundary conditions. The Neumann boundary condition is appropriate, for example, as the no-flux boundary condition, whereas the Dirichlet boundary condition is necessary when the diffusion equation is solved with a fixed concentration on the boundaries. For Fick's second law of diffusion, the original governing equation is expressed as

$$
\frac{\partial C}{\partial t}=-\nabla \cdot \vec{j}+S=\nabla \cdot(D \nabla C)+S
$$

where $\vec{j}$ is the flux vector, $D$ is the diffusion coefficient, $C$ is the concentration, $S$ is the source term, and $t$ is the time. Instead of directly solving the diffusion equation, we multiply equation (3) by $\psi$, the domain parameter that describes the domain in which diffusion occurs, and use the identity $\psi \nabla \cdot(D \nabla C)=\nabla \cdot(\psi D \nabla C)-\nabla \psi \cdot(D \nabla C)$ to obtain the SBM formulated diffusion equation:

$$
\psi \frac{\partial C}{\partial t}=\nabla \cdot(\psi D \nabla C)-\nabla \psi \cdot(D \nabla C)+\psi S
$$

Next, we consider the boundary condition in this formulation. The Neumann boundary condition is the inward flux across the domain boundary, mathematically the normal gradient of $C$ at the diffuse interface, and is treated as

$$
D B_{\mathrm{N}} \equiv D \frac{\partial C}{\partial n} \equiv \vec{n} \cdot \vec{j}=-\frac{\nabla \psi}{|\nabla \psi|} \cdot(D \nabla C),
$$

where $\vec{n}=\nabla \psi /|\nabla \psi|$ is the unit inward normal vector at the boundary defined in the diffuse interface description. Note that the flux at the interface is equal to $D B_{\mathrm{N}}$. Equation (5) is rearranged to become $\nabla \psi \cdot(D \nabla C)=-|\nabla \psi| D B_{\mathrm{N}}$ and substituted back into equation (4); thus, we obtain

$$
\frac{\partial C}{\partial t}=\frac{1}{\psi} \nabla \cdot(\psi D \nabla C)+\frac{|\nabla \psi|}{\psi} D B_{\mathrm{N}}+S,
$$

with the Neumann boundary condition appearing in the second term. When a no-flux boundary is imposed, the second term vanishes and the resulting equation is the same as that proposed in $[1-3,22,24]$.

To impose the Dirichlet boundary condition, we manipulate the original governing equation in a procedure similar to the derivation of equation (6). Multiplying equation (4) by $\psi$ and using the identity $\psi \nabla \psi \cdot(D \nabla C)=D[\nabla \psi \cdot \nabla(\psi C)-C \nabla \psi \cdot \nabla \psi]=D[\nabla \psi$. $\left.\nabla(\psi C)-C|\nabla \psi|^{2}\right]$ to replace the second term, we obtain

$$
\frac{\partial C}{\partial t}=\frac{1}{\psi} \nabla \cdot(\psi D \nabla C)-\frac{1}{\psi^{2}} D\left[\nabla \psi \cdot \nabla(\psi C)-B_{\mathrm{D}}|\nabla \psi|^{2}\right]+S,
$$

where $B_{\mathrm{D}}$ is the Dirichlet boundary condition imposed at the diffuse interface to replace $C$, associated with $|\nabla \psi|^{2}$ in the third term. The convergence to the imposed Neumann and Dirichlet boundary conditions is shown in appendix A. 
In this method, the value of gradient at boundary, $B_{\mathrm{N}}$, and the value at boundary, $B_{\mathrm{D}}$, are not specified to be constant values; they can vary spatially and/or temporally or be functions of $C$ or other parameters. In addition, it is convenient to use weighting factors to combine equations (6) and (7) to impose Neumann and Dirichlet boundary conditions simultaneously to yield mixed (or Robin) boundary conditions. The equation then becomes

$\frac{\partial C}{\partial t}=\frac{1}{\psi} \nabla \cdot(\psi D \nabla C)+\frac{|\nabla \psi|}{\psi} D B_{\mathrm{N}} W_{\mathrm{N}}-\frac{D}{\psi^{2}}\left[\nabla \psi \cdot \nabla(\psi C)-B_{\mathrm{D}}|\nabla \psi|^{2}\right] W_{\mathrm{D}}+S$,

where $W_{\mathrm{N}}$ and $W_{\mathrm{D}}$ are the spatially dependent weighting factors for the Neumann and Dirichlet boundary conditions, respectively $\left(W_{\mathrm{N}}+W_{\mathrm{D}}=1\right)$. These factors can be a linear combination when imposing Robin boundary conditions or be employed to impose Neumann and Dirichlet boundary conditions at different regions of the interface. Moreover, a small nonzero value $\left(10^{-16}<v<10^{-6}\right)$ should be added to the domain parameter appearing in the denominators to avoid singularities resulting from the terms $1 / \psi$ and $1 / \psi^{2}$ in regions where $\psi=0$.

\subsection{Coupled surface-bulk diffusion equation}

The second example demonstrates that surface diffusion can be incorporated into the SBM equation derived above. For this case, we take the set of equations that includes the surface reaction, surface diffusion and bulk diffusion to describe an oxygen reduction model in a solid oxide fuel cell (SOFC) cathode [42]. A brief description of the problem is given in section 5.1. The oxygen-vacancy concentration, $C$, on the cathode surface is governed by Fick's second law for surface diffusion:

$$
-D_{\mathrm{b}} \frac{\partial C}{\partial n}=\kappa C-D_{\mathrm{s}} \nabla_{\mathrm{s}}^{2} C+L \frac{\partial C}{\partial t}
$$

where $n$ is the coordinate along the inward unit normal vector of the surface and $\nabla_{\mathrm{s}}^{2}$ is the surface Laplacian. The parameters $D_{\mathrm{b}}, \kappa, D_{\mathrm{s}}$ and $L$ are the bulk diffusivity, reaction rate, surface diffusivity and accumulation coefficient, respectively. Thus, the term on the left-hand side represents the flux from the bulk, and the terms on the right-hand side represent the surface reaction, surface diffusion and surface concentration accumulation, respectively [42]. Here, these parameters are all assumed to be constant for simplicity. In the bulk of the cathode material, the oxygen-vacancy diffusion is governed by Fick's second law for bulk diffusion:

$$
\frac{\partial C}{\partial t}=D_{\mathrm{b}} \nabla^{2} C
$$

To simulate the oxygen-vacancy concentration evolution in the cathode, the two diffusion equations, (9) and (10), are coupled and must be solved simultaneously, with the flux normal to the cathode surface as a common boundary condition. Recently, a similar set of equations was formulated using another diffuse-interface approach combined with an asymptotic analysis [32], which led to two differential equations coupled by a common boundary condition. We show below that we can eliminate the need for solving two separate equations by applying the SBM formulation described herein to obtain a single equation that governs both surface and bulk effects.

Similar to the derivation of equation (6), we first multiply equation (10) by $\psi$ and apply the product rule of differentiation to obtain the bulk diffusion equation containing a boundary term, $D_{\mathrm{b}} \nabla \psi \cdot \nabla C$, similar to equation (4). As in equation (5), the normal gradient at the diffuse interface is defined by $\partial C / \partial n=-\nabla C \cdot \nabla \psi /|\nabla \psi|$. Substituting this relationship back into equation (9) and rearranging terms gives

$$
\nabla \psi \cdot \nabla C=\frac{|\nabla \psi|}{D_{\mathrm{b}}}\left[\kappa C-D_{\mathrm{s}} \nabla_{\mathrm{s}}^{2} C+L \frac{\partial C}{\partial t}\right] .
$$


Using this relation, we replace the boundary term to obtain the SBM formulated equation:

$$
\frac{\partial C}{\partial t}=\frac{1}{\psi} D_{\mathrm{b}} \nabla \cdot(\psi \nabla C)-\frac{|\nabla \psi|}{\psi}\left[\kappa C-D_{\mathrm{s}} \nabla_{\mathrm{s}}^{2} C+L \frac{\partial C}{\partial t}\right],
$$

which combines the bulk diffusion and surface diffusion terms into a single equation used in the examples presented in sections 4.2 and 5.1. Again, a small nonzero value should be added to the domain parameter appearing in the denominators. In the bulk $(|\nabla \psi|=0$ and $\psi=1)$, equation (12) reduces to equation (10). When the interfacial thickness approaches zero, it reduces to equation (9) at the interface $(|\nabla \psi| \neq 0)$, as proven in appendix A.

The surface Laplacian $\left(\nabla_{\mathrm{s}}^{2}=\nabla_{\mathrm{s}} \cdot \nabla_{\mathrm{s}}\right)$ is calculated according to the surface gradient given by

$$
\nabla_{\mathrm{s}}=(\boldsymbol{I}-\boldsymbol{n} \otimes \boldsymbol{n}) \nabla=\left(\boldsymbol{I}-\frac{\nabla \psi}{|\nabla \psi|} \otimes \frac{\nabla \psi}{|\nabla \psi|}\right) \nabla,
$$

where $\boldsymbol{I}$ is the unity tensor, ' $\otimes$ ' is the dyadic product, and $\boldsymbol{n}$ is the inward unit normal vector of the diffuse interface, as used in [32]. In indicial notation, the surface gradient is expressed as $\left(\delta_{i j}-n_{i} n_{j}\right) \partial / \partial x_{j}$, where $\delta_{i j}$ is the Kronecker delta $\left(\delta_{i j}=1\right.$ if $i=j$, and $\delta_{i j}=0$ if $\left.i \neq j\right)$. The repeated indices indicate summation over the index. See appendix B for details. To simulate only surface diffusion on a diffuse-interface-described geometry, one can simply eliminate the bulk-related and reaction terms in equation (9) to obtain $L(\partial C / \partial t)=D_{\mathrm{s}} \nabla_{\mathrm{s}}^{2} C$, such that the concentration evolves only over the interfacial region.

\subsection{Mechanical equilibrium equation}

The SBM can also be applied to the mechanical equilibrium equation. When a solid body is in mechanical equilibrium, all forces acting on the body are balanced in all directions, as represented by $\partial \sigma_{i j} / \partial x_{j}=0$, where $\sigma_{i j}$ is a stress tensor component, which is the force per unit area along $j$ th axis on the surface whose normal vector is along the $i$ th axis. Repeated indices indicate summation over the index. For linear elasticity, the stress tensor is given by the generalized form of Hooke's law: $\sigma_{i j}=C_{i j k l}\left(\varepsilon_{k l}-\rho \delta_{k l}\right)$, where $C_{i j k l}$ is the elastic constant tensor and $\rho$ is a scalar source of body force, such as thermal expansion $(\alpha \Delta T)$ or misfit eigenstrain $\left(\left(a_{p}-a_{m}\right) / a_{m}\right.$, where $a_{p}$ and $a_{m}$ are the lattice constants of the precipitate and matrix phases, respectively), depending on the governing physics. The total strain tensor is defined by the gradients of displacements as $\varepsilon_{i j}=\left[\left(\partial u_{i} / \partial x_{j}\right)+\left(\partial u_{j} / \partial x_{i}\right)\right] / 2$, where $u_{i}$ is the infinitesimal displacement in the $i$ th direction. Substituting Hooke's law and the total strain back into the mechanical equilibrium equation gives

$$
\frac{\partial}{\partial x_{j}} C_{i j k l} \frac{1}{2}\left(\frac{\partial u_{k}}{\partial x_{l}}+\frac{\partial u_{l}}{\partial x_{k}}\right)=\frac{\partial}{\partial x_{j}}\left(\rho C_{i j k l} \delta_{k l}\right) .
$$

Multiplying equation (14) by the domain parameter that distinguishes the elastic solid region $(\psi=1)$ from the environment $(\psi=0)$ and using the product rule of differentiation yields the SBM formulation:

$$
\begin{gathered}
\frac{\partial}{\partial x_{j}}\left[\psi C_{i j k l} \frac{1}{2}\left(\frac{\partial u_{k}}{\partial x_{l}}+\frac{\partial u_{l}}{\partial x_{k}}\right)\right]-\left(\frac{\partial \psi}{\partial x_{j}}\right)\left\{C_{i j k l}\left[\frac{1}{2}\left(\frac{\partial u_{k}}{\partial x_{l}}+\frac{\partial u_{l}}{\partial x_{k}}\right)-\rho \delta_{k l}\right]\right\} \\
=\frac{\partial}{\partial x_{j}}\left(\psi \rho C_{i j k l} \delta_{k l}\right)
\end{gathered}
$$

see appendix $\mathrm{C}$ for details of the derivation. 
The traction exerted on the solid surface is defined by $N_{i}=-\sigma_{i j} n_{j}$, where $n_{j}=\nabla \psi /|\nabla \psi|$ is the inward unit normal of the solid surface. (In indicial notation, $\partial \psi / \partial x_{i}=\nabla \psi$ and $\left.\sqrt{\left(\partial \psi / \partial x_{i}\right)\left(\partial \psi / \partial x_{i}\right)}=|\nabla \psi|.\right)$ Therefore, the surface traction force is given by

$$
N_{i}=-\left\{C_{i j k l}\left[\frac{1}{2}\left(\frac{\partial u_{k}}{\partial x_{l}}+\frac{\partial u_{l}}{\partial x_{k}}\right)-\rho \delta_{k l}\right]\right\}\left(\frac{\nabla \psi}{|\nabla \psi|}\right) .
$$

Substituting equation (16) into equation (15) yields the SBM formulated mechanical equilibrium equation with a traction boundary condition on the solid surface:

$$
\frac{\partial}{\partial x_{j}}\left[\psi C_{i j k l} \frac{1}{2}\left(\frac{\partial u_{k}}{\partial x_{l}}+\frac{\partial u_{l}}{\partial x_{k}}\right)\right]+|\nabla \psi| N_{i}=\frac{\partial}{\partial x_{j}}\left(\psi \rho C_{i j k l} \delta_{k l}\right),
$$

where $\partial\left(\psi \rho C_{i j k l} \delta_{k l}\right) / \partial x_{j}=\tilde{\rho}_{i}$ can be treated as an effective body force in the $i$ th direction.

For linear elasticity problems with prescribed displacements at the solid surface, one can perform the SBM formulation, as in the derivation of the Dirichlet boundary condition in section 3.2, by multiplying equation (14) by $\psi^{2}$ and using the product rule to obtain

$$
\begin{aligned}
\psi \frac{\partial}{\partial x_{j}}\left[\psi C_{i j k l}\right. & \left.\frac{1}{2}\left(\frac{\partial u_{k}}{\partial x_{l}}+\frac{\partial u_{l}}{\partial x_{k}}\right)\right]-\left\{\left(\frac{\partial \psi}{\partial x_{j}}\right)\left[C_{i j k l} \frac{1}{2}\left(\frac{\partial \psi u_{k}}{\partial x_{l}}+\frac{\partial \psi u_{l}}{\partial x_{k}}\right)\right]\right. \\
& \left.-\left(\frac{\partial \psi}{\partial x_{j}}\right) C_{i j k l} \frac{1}{2}\left(u_{k} \frac{\partial \psi}{\partial x_{l}}+u_{l} \frac{\partial \psi}{\partial x_{k}}\right)\right\}=\psi^{2} \frac{\partial}{\partial x_{j}}\left(\rho C_{i j k l} \delta_{k l}\right),
\end{aligned}
$$

where the displacements $u_{k}$ and $u_{l}$ appearing in the third term on the left-hand side should be the boundary values of the displacements at the solid surface; see appendix $C$ for the derivation. A similar formulation for solving the mechanical equilibrium equation within a domain defined by a phase-field-like order parameter can also be obtained by the asymptotic approach by matching terms of different orders [43].

\subsection{Equations for phase transformations with additional boundaries}

Phase transformations affected by a mobile or immobile surface or other boundaries are of importance in many materials processes, including heterogeneous nucleation that occurs at material interfaces $[19,20]$. Maintaining a proper contact angle at the three-phase boundary (where the interface between the two phases meets the surface) is necessary to capture the dynamics accurately because the contact angle represents the difference between the surface energies (tensions) of the different phase boundaries. Although researchers have previously developed methods for imposing contact-angle boundary conditions on sharp domain walls $[19,20]$, here we show that a similar model with diffuse domain walls can be obtained simply by applying the approach described above. Below, we assume that the boundary is immobile, but this assumption can be easily removed by describing the evolution of the domain parameter as dictated by the physics of the system.

In the Allen-Cahn and Cahn-Hilliard equations of the phase-field model, the total free energy has the following form [36-38]:

$$
F=\int_{\Omega}\left[f(\phi)+\frac{\epsilon^{2}}{2}|\nabla \phi|^{2}\right] \mathrm{d} \Omega,
$$

where $\phi$ is the phase-field order parameter commonly used to define different phases, $f(\phi)$ is a double-well free energy functional (in terms of $\phi), \epsilon$ is the gradient energy coefficient, and $\Omega$ is the domain of interest. At the extremum of the functional $F$, the variational derivative of the total free energy vanishes: $\delta F / \delta \phi=0$. This requirement provides the following conditions: $\partial f / \partial \phi-\epsilon^{2} \nabla^{2} \phi=0 \in \Omega$, which can be reformulated as $\nabla f=\nabla\left(\epsilon^{2}|\nabla \phi|^{2}\right) / 2$, 
by multiplying both sides by $\nabla \phi$. We thus find a useful equality for deriving the contact angle boundary condition: $|\nabla \phi|=\sqrt{2 f} / \epsilon$; see appendix D for details.

In the SBM, we introduce a domain parameter $\psi$ to incorporate boundary conditions into the original governing equation. As mentioned previously, the level sets of this domain parameter $\psi$ describe the diffuse boundaries and should satisfy $\vec{n}=\nabla \psi /|\nabla \psi|$. On $\partial \Omega$, a contact angle, $\theta$, forms such that $\vec{n} \cdot(\nabla \phi /|\nabla \phi|)=-\cos \theta$, where $\nabla \phi /|\nabla \phi|$ is the unit normal vector of the phase interface (pointing to regions where $\phi=1$ ). We can thus derive the following equation for the boundary condition:

$$
\nabla \psi \cdot \nabla \phi=-|\nabla \psi| \cos \theta \frac{\sqrt{2 f}}{\epsilon} .
$$

This contact-angle boundary condition is similar to that suggested by Warren et al [20] for contacting a sharp interface, in which a Dirac delta function replaces $|\nabla \psi|$.

The chemical potential that drives the morphological evolution is defined by the variational derivative of the total free energy of the system: $\mu=\delta F / \delta \phi=\partial f / \partial \phi-\epsilon^{2} \nabla^{2} \phi$. We can apply the SBM formulation to the chemical potential by multiplying it by the domain parameter $\psi$ and applying the product rule to obtain

$\mu=\frac{\partial f}{\partial \phi}-\frac{\epsilon^{2}}{\psi} \nabla \cdot(\psi \nabla \phi)+\frac{\epsilon^{2}}{\psi} \nabla \psi \cdot \nabla \phi=\frac{\partial f}{\partial \phi}-\frac{\epsilon^{2}}{\psi} \nabla \cdot(\psi \nabla \phi)-\frac{\epsilon|\nabla \psi|}{\psi} \sqrt{2 f} \cos \theta$,

where equation (20) was used in the third term.

For a nonconserved order parameter in the phase-field models, the evolution is governed by the Allen-Cahn equation [38] (also known as the time-dependent Ginzburg-Landau equation [39]), in which the order parameter evolves according to the local chemical potential variation:

$$
\frac{\partial \phi}{\partial t}=-M \mu=-M\left(\frac{\partial f}{\partial \phi}-\frac{\epsilon^{2}}{\psi} \nabla \cdot(\psi \nabla \phi)-\frac{\epsilon|\nabla \psi|}{\psi} \sqrt{2 f} \cos \theta_{\mathrm{B}}\right),
$$

where $M$ is the mobility coefficient and $\theta_{\mathrm{B}}$ is the contact angle boundary condition imposed at the three-phase boundary.

For a conserved order parameter, its evolution is governed by the Cahn-Hilliard equation, where the rate of the order parameter change is equal to the divergence of its flux, which is proportional to the gradient of the chemical potential [36,37], $\partial \phi / \partial t=\nabla \cdot(M \nabla \mu)$. The SBM formulation (derived in a similar manner as in the derivation in section 3.2) is given by $\psi(\partial \phi / \partial t)=\nabla \cdot(\psi M \nabla \mu)-\nabla \psi \cdot(M \nabla \mu)$. Note that $-M \nabla \mu=\vec{j}$ is the flux of the conserved order parameter; thus, the second term represents the fluxes normal to the domain boundary (equivalent to equation (5)). The SBM formulation of the Cahn-Hilliard equation is thus written as

$$
\frac{\partial \phi}{\partial t}=\frac{1}{\psi} \nabla \cdot\left[\psi M \nabla\left(\frac{\partial f}{\partial \phi}-\frac{\epsilon^{2}}{\psi} \nabla \cdot(\psi \nabla \phi)-\frac{\epsilon|\nabla \psi|}{\psi} \sqrt{2 f} \cos \theta_{\mathrm{B}}\right)\right]+\frac{|\nabla \psi|}{\psi} J_{n},
$$

where $J_{n}=\vec{n} \cdot \vec{j}$. For a closed system, $J_{n}$ is zero. Note that a small nonzero value should to be added to the domain parameter in the denominators in equations (22) and (23) to avoid division by zero.

\section{Validation of the presented approach}

Parametric studies were conducted to examine how the error depends on the two dimensionless parameters: the ratio of characteristic length to interfacial thickness and the ratio of interfacial thickness to grid spacing. The former can be viewed as the characteristic length in the unit of the interfacial thickness, and the latter is the number of the grid points within the interface. 
We performed computationally inexpensive one-dimensional (1D) simulations to explore the parameter space as detailed in section 4.1. With the general error behavior characterized in the 1D simulations, we focused on the effect of domain-interface thickness in the more complicated two-dimensional (2D) problems in sections 4.2 and 4.3.

\subsection{D diffusion equation}

We conducted a 1D simulation to demonstrate that the Neumann and Dirichlet boundary conditions were satisfied on two different sides of the domain. Because the introduction of smoothed boundary results additional errors beyond the usual numerical errors, we attempt here to quantify and characterize the errors associated with the formulation. Fick's second diffusion equation with given source and sink terms was solved within the domain defined by $\psi=1$. The diffusion coefficient was set at 1 , and the source and sink strengths were 0.02 and 0.01 , respectively. On the right boundary of the diffusion domain, the gradient of $C$ was set at -0.1 , and on the left boundary, the value of $C$ was set at 0.4 . We selected the 1D computational box for $0<x<30$ and used a hyperbolic tangent function for the continuous domain parameter $\psi$ :

$$
\psi=\frac{1}{2}\left[\tanh \left(\frac{x-5}{\zeta}\right)-\tanh \left(\frac{x-25}{\zeta}\right)\right],
$$

where $\zeta$ is the coefficient for adjusting the interfacial thickness. The left and right interfaces are located at $x=5$ and $x=25$, respectively. The specific value of the domain size is not important here because we will examine the ratio between domain size and interfacial thickness to investigate the error behavior in the parametric studies presented later. The interfacial thickness is given approximately by $\xi_{0}=4.185 \zeta$ where the interfacial region is defined by the range $0.015<\psi<0.985$. We applied the SBM formulation, as in the derivation of equation (8), to reformulate the original diffusion equation,

$$
\frac{\partial C}{\partial t}=\frac{\partial^{2} C}{\partial x^{2}}-\frac{C}{0.01}+0.02
$$

to

$$
\begin{aligned}
\frac{\partial C}{\partial t}=\frac{1}{\psi} \frac{\partial}{\partial x}\left(\psi \frac{\partial C}{\partial x}\right)-\frac{0.1}{\psi}\left|\frac{\partial \psi}{\partial x}\right| H_{\mathrm{V}}\left(l_{\mathrm{m}}\right)-\frac{1}{\psi^{2}}\left[\frac{\partial \psi}{\partial x} \frac{\partial(\psi C)}{\partial x}-0.4\left|\frac{\partial \psi}{\partial x}\right|^{2}\right]\left[1-H_{\mathrm{V}}\left(l_{\mathrm{m}}\right)\right] \\
-\frac{C}{0.01}+0.02
\end{aligned}
$$

where $H_{\mathrm{V}}\left(l_{\mathrm{m}}\right)$ is the Heaviside function used to specify the choice of the boundary condition and $l_{\mathrm{m}}=15$ is the midpoint of the diffusion domain. Therefore, the second and third terms only apply to the right and left interfaces, respectively. The initial concentration was $C=0$ everywhere in the computational box. A standard central finite difference scheme in space and an Euler explicit time scheme were employed in the simulations.

Figure 2 shows the concentration profiles recorded at four different times (solid blue lines). The domain parameter is plotted in the red line (the red open circles indicate the positions of grid points). The computational box was discretized to 1200 grid points $\left(\Delta x=2.5 \times 10^{-2}\right)$, and $\zeta$ was taken to be $2.87 \times 10^{-2}$, such that the interfacial thickness is approximately $\xi_{0}=0.12=4.80 \Delta x$. The parameters are given as case 1(b) in table 1. On the right interface, it can be clearly observed that $\mathrm{d} C / \mathrm{d} x=-0.1$ at all times (except for a rapid change from $\mathrm{d} C / \mathrm{d} x=0$ to $\mathrm{d} C / \mathrm{d} x=-0.1$ in the very early transient period). In the early period, the concentration even took negative values to satisfy the gradient boundary condition imposed at the right interface. In contrast, the concentration remained at 0.4 at the left interface 


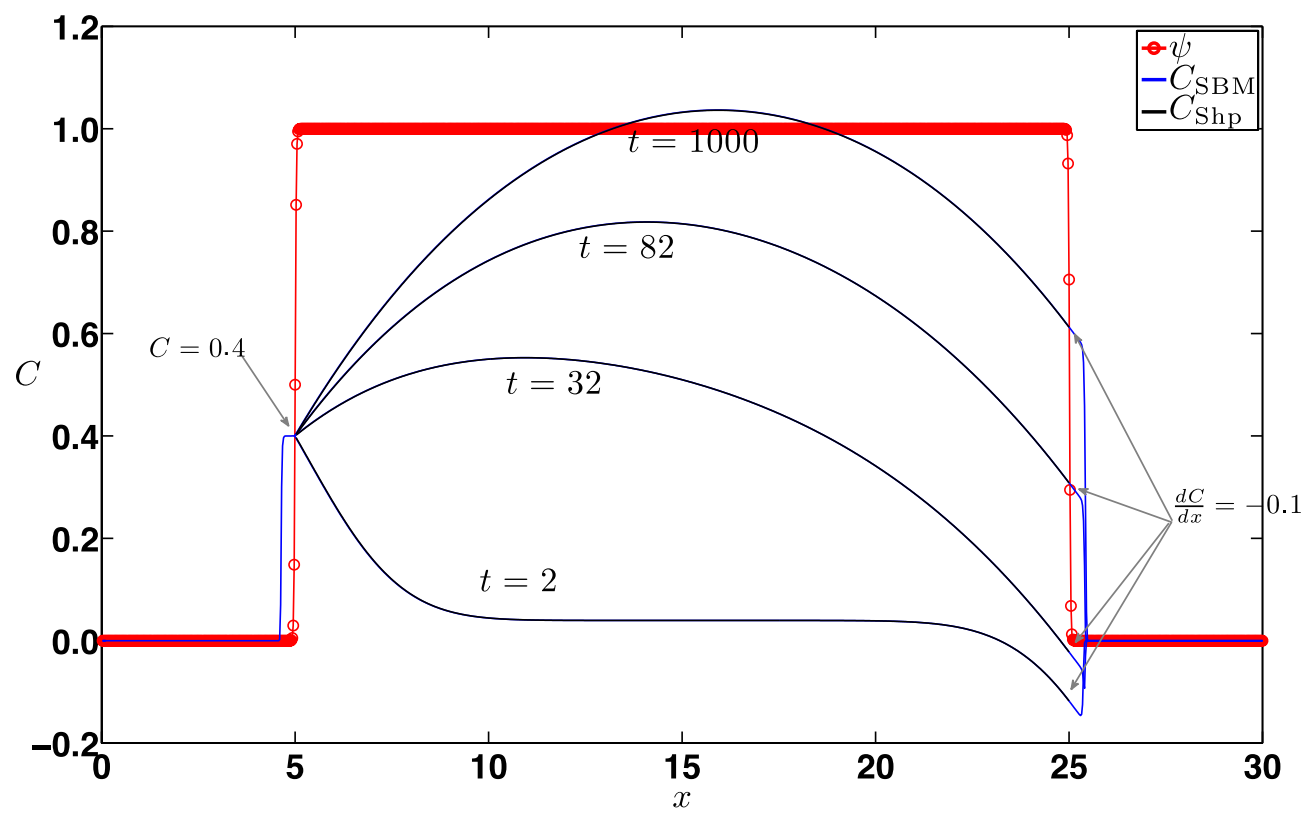

Figure 2. Demonstration of the SBM on the $1 \mathrm{D}$ diffusion equation. The red line with open circles is the domain parameter, and the blue lines are the concentration profiles at different times. The Neumann and Dirichlet boundary conditions are imposed at the right and left boundaries, respectively. The black lines are the corresponding solutions of the sharp-interface version of the diffusion equation.

during the entire diffusion process (except in the very early transient period, during which $C$ changed from 0 to 0.4 ). For comparison, we solved the original sharp-interface diffusion equation (25) with the Neumann and Dirichlet boundary conditions imposed at $x=5$ and $x=25$, respectively. The same spatial and temporal discretization schemes were employed. By comparing two resolutions, $\Delta x=2.5 \times 10^{-2}$ and $\Delta x=1.25 \times 10^{-2}$, we ensured that the sharp-interface result was converged sufficiently to the exact solution. Here, we selected the result for $\Delta x=2.5 \times 10^{-2}$ as the reference numerical solution for analyzing the errors resulting from the SBM formulation since it has the same resolution as the SBM calculation. The comparison shows an excellent agreement between the two methods. This clearly demonstrates that both Neumann and Dirichlet boundary conditions are satisfied on the diffuse interfaces, and the SBM formulated equation reproduces the same result to the corresponding sharp-interface version.

As mentioned earlier, there are two dimensionless parameters that influence the error in the calculations. One is the characteristic-length-to-interface ratio, $\mathcal{R}_{1}=L_{\mathrm{c}} /\left(2 \xi_{0}\right)$, which indicates how large the characteristic length in the system (e.g. the domain size), $L_{\mathrm{c}}$, is compared with the interfacial thickness. This is equivalent to the characteristic length in the unit of the interfacial thickness. As an example, if the domain size is not sufficiently large compared with the domain-interface thickness, the bulk portion of the domain will not be represented accurately. If multiple characteristic lengths are present in the system, the smallest value should be considered. Since we focus on the intermediate to later stage of diffusion in this 1D study, the characteristic length is the size of the domain where diffusion occurs. Note that the factor of 2 in $\mathcal{R}_{1}$ emerges because two interfaces are present on the two sides of the domain. The second parameter is the interface-to-grid-size ratio, $\mathcal{R}_{2}=\xi_{0} / \Delta x$, 
Table 1. Relative errors, $e$, for the 1D SBM diffusion equation to the sharp-interface solution with various parameters. Each of the markers, $*, \diamond$ and $\triangleleft$, denotes the identical result from a set of parameters.

\begin{tabular}{|c|c|c|c|c|c|c|c|c|c|c|}
\hline \multirow[t]{3}{*}{ Case } & \multicolumn{5}{|c|}{1} & \multicolumn{5}{|c|}{2} \\
\hline & \multicolumn{5}{|c|}{$\begin{aligned} \Delta x & =2.5 \times 10^{-2} \\
v & =1 \times 10^{-7}\end{aligned}$} & \multicolumn{5}{|c|}{$\begin{array}{l}\zeta=1.145 \Delta x \\
v=1 \times 10^{-7}\end{array}$} \\
\hline & $\zeta$ & $\xi_{0}$ & $\mathcal{R}_{1}$ & $\mathcal{R}_{2}$ & $e$ & $\Delta x$ & $\xi_{0}$ & $\mathcal{R}_{1}$ & $\mathcal{R}_{2}$ & $e$ \\
\hline (a) & $1.43 \times 10^{-2}$ & 0.06 & 166.67 & 2.40 & $2.74 \times 10^{-4}$ & $1.25 \times 10^{-2}$ & 0.06 & 166.67 & 4.80 & $3.93 \times 10^{-4}$ \\
\hline (b) & $2.87 \times 10^{-2}$ & 0.12 & 83.33 & 4.80 & $* 7.88 \times 10^{-4}$ & $2.50 \times 10^{-2}$ & 0.12 & 83.33 & 4.80 & $* 7.88 \times 10^{-4}$ \\
\hline (c) & $5.73 \times 10^{-2}$ & 0.24 & 41.67 & 9.60 & $\diamond 1.72 \times 10^{-3}$ & $5.00 \times 10^{-2}$ & 0.24 & 41.67 & 4.80 & ${ }^{\triangleleft} 1.58 \times 10^{-3}$ \\
\hline (d) & $1.15 \times 10^{-1}$ & 0.48 & 20.83 & 19.2 & $3.53 \times 10^{-3}$ & $1.00 \times 10^{-1}$ & 0.48 & 20.83 & 4.80 & $3.20 \times 10^{-3}$ \\
\hline (e) & $2.29 \times 10^{-1}$ & 0.96 & 10.42 & 38.4 & $7.20 \times 10^{-3}$ & $2.00 \times 10^{-1}$ & 0.96 & 10.42 & 4.80 & $6.54 \times 10^{-3}$ \\
\hline (f) & $4.59 \times 10^{-1}$ & 1.92 & 5.21 & 76.8 & $1.49 \times 10^{-2}$ & $4.00 \times 10^{-1}$ & 1.92 & 5.21 & 4.80 & $1.39 \times 10^{-2}$ \\
\hline \multirow[t]{3}{*}{ Case } & \multicolumn{5}{|c|}{3} & \multicolumn{5}{|c|}{4} \\
\hline & \multicolumn{5}{|c|}{$\begin{array}{c}\zeta=5.73 \times 10^{-2} \\
v=1 \times 10^{-7}\end{array}$} & \multicolumn{5}{|c|}{$\begin{array}{l}\zeta=2.87 \times 10^{-2} \\
\Delta x=2.5 \times 10^{-2}\end{array}$} \\
\hline & $\Delta x$ & $\xi_{0}$ & $\mathcal{R}_{1}$ & $\mathcal{R}_{2}$ & $e$ & $v$ & $\xi_{0}$ & $\mathcal{R}_{1}$ & $\mathcal{R}_{2}$ & $e$ \\
\hline (a) & $1.25 \times 10^{-2}$ & 0.24 & 41.67 & 19.2 & $1.75 \times 10^{-3}$ & $1.0 \times 10^{-2}$ & 0.12 & 83.33 & 4.80 & $7.75 \times 10^{-3}$ \\
\hline (b) & $2.50 \times 10^{-2}$ & 0.24 & 41.67 & 9.60 & $\diamond 1.72 \times 10^{-3}$ & $1.0 \times 10^{-3}$ & 0.12 & 83.33 & 4.80 & $1.39 \times 10^{-3}$ \\
\hline (c) & $5.00 \times 10^{-2}$ & 0.24 & 41.67 & 4.80 & ${ }^{\triangleleft} 1.58 \times 10^{-3}$ & $1.0 \times 10^{-5}$ & 0.12 & 83.33 & 4.80 & $7.93 \times 10^{-4}$ \\
\hline (d) & $1.00 \times 10^{-1}$ & 0.24 & 41.67 & 2.40 & $1.16 \times 10^{-3}$ & $1.0 \times 10^{-7}$ & 0.12 & 83.33 & 4.80 & $* 7.88 \times 10^{-4}$ \\
\hline (e) & $2.00 \times 10^{-1}$ & 0.24 & 41.67 & 1.20 & $7.53 \times 10^{-4}$ & $1.0 \times 10^{-9}$ & 0.12 & 83.33 & 4.80 & $7.88 \times 10^{-4}$ \\
\hline (f) & $4.00 \times 10^{-1}$ & 0.24 & 41.67 & 0.60 & Unstable & $1.0 \times 10^{-11}$ & 0.12 & 83.33 & 4.80 & $7.88 \times 10^{-4}$ \\
\hline
\end{tabular}

which indicates how large the interface is compared with the grid size. This is equivalent to the number of grid points within the interfacial region, and if it is very small, the numerical accuracy may not be sufficient. Various simulations were conducted for parametric studies to analyze the effects of the two ratios in the SBM. As will be seen, the numerical error is insensitive on $\mathcal{R}_{2}$ in $1 \mathrm{D}$ calculations, but it becomes important in $2 \mathrm{D}$ or $3 \mathrm{D}$ calculations where curved and tilted interfaces must be smoothly represented.

To control $\mathcal{R}_{1}$ and $\mathcal{R}_{2}$, we modified $\zeta$ (equivalent to varying $\xi_{0}$ ) and $\Delta x$ while fixing the immersed domain size as $L_{\mathrm{c}}=20$. The relative errors were calculated for sets of the two ratios, and the values at steady state are presented in table 1 . Here, the relative error in the concentration is defined by the root-mean-square ( $\mathrm{rms}$ ) deviation between the SBM result and the sharp-interface solution divided by the average sharp-interface result. The examination of the tabulated values provides estimation of errors in the SBM calculations and can be used as a guideline for setting parameters in other calculations. We consider three cases: (1) changing the interfacial thickness while holding other quantities, (2) changing $\mathcal{R}_{1}$ while fixing $\mathcal{R}_{2}$ and (3) changing $\mathcal{R}_{2}$ while fixing $\mathcal{R}_{1}$. Finally, we examine the effect of the small parameter used to regularize the singularity $\left(1 / \psi\right.$ and $1 / \psi^{2}$ in equation (26)).

In the first set of parametric study (case 1), we simply varied the interfacial thickness by selecting several values of $\zeta$ in equation (24) while the grid size was kept at $\Delta x=2.5 \times 10^{-2}$, as in case 1 in table 1 . As a result, both $\mathcal{R}_{1}$ and $\mathcal{R}_{2}$ were modified simultaneously in this case. Such a case arises often when experimentally reconstructed 3D microstructures are used as an input. These structures have voxels with predetermined physical sizes, and interfaces are typically sharp (i.e. a step function describes multiple phases). In order to input such structures, 


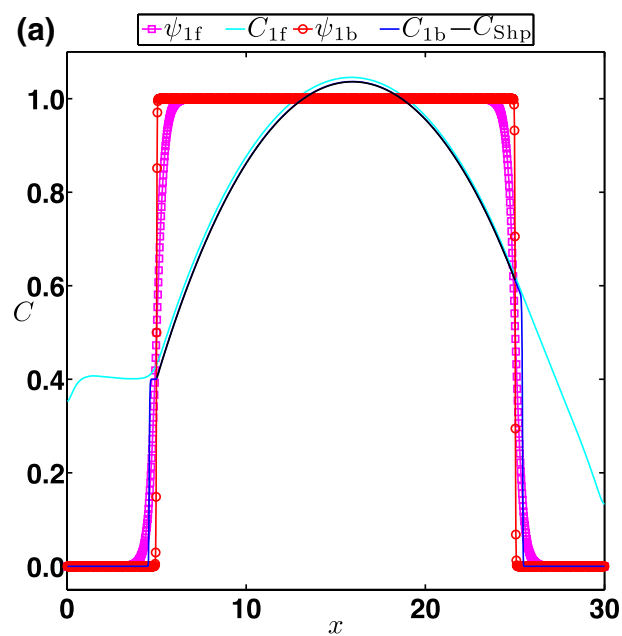

(b) $-e_{1 \mathrm{a}}-e_{1 \mathrm{~b}}-e_{1 \mathrm{c}}-e_{1 \mathrm{~d}}-e_{1 \mathrm{e}}-e_{1 \mathrm{f}}$
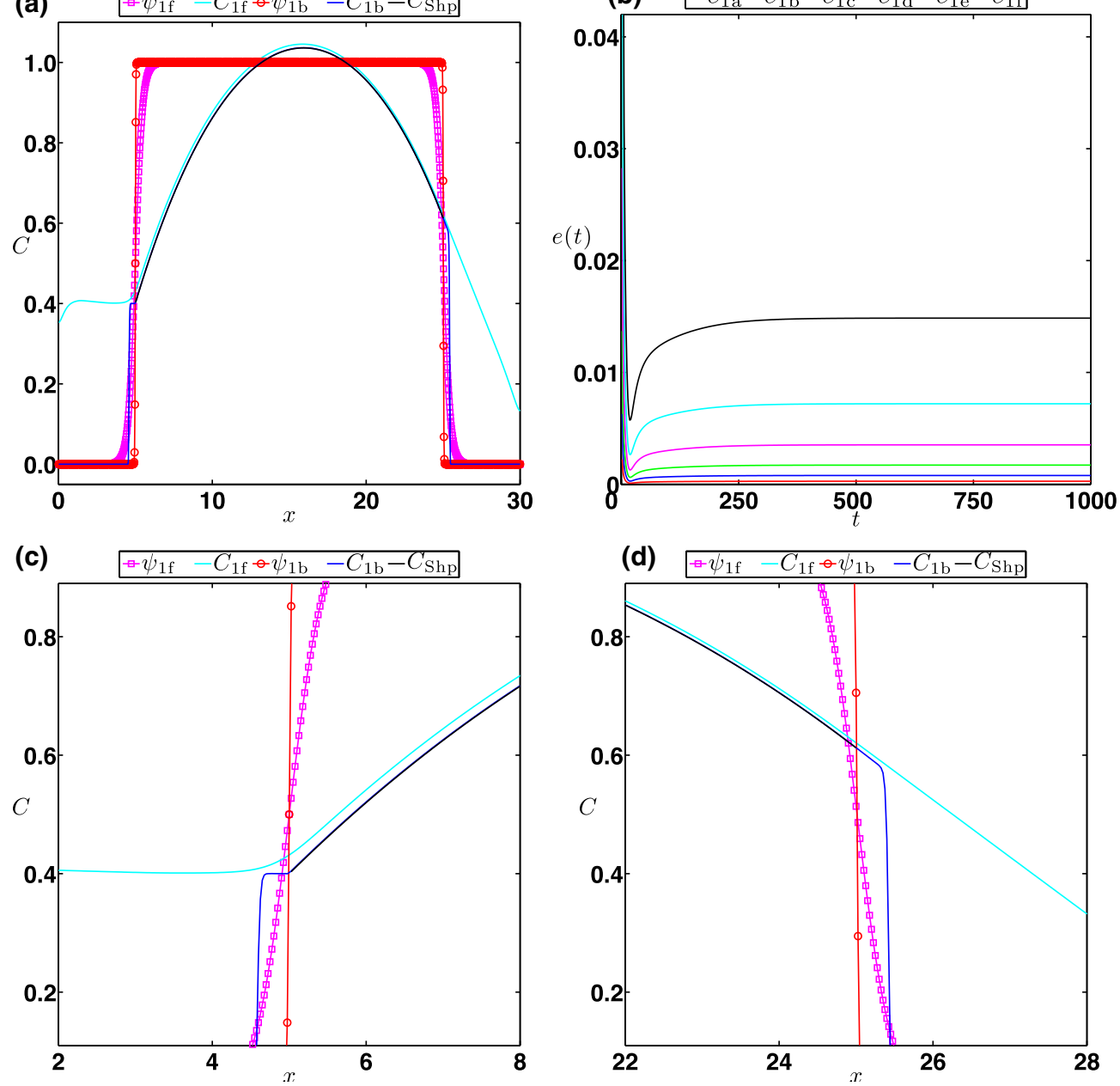

Figure 3. (a) Equilibrium concentrations in domains of two different interfacial thicknesses, corresponding to case 1 (f) $\left(\zeta=4.59 \times 10^{-1}\right)$ and case $1(\mathrm{~b})\left(\zeta=2.87 \times 10^{-2}\right)$ in table 1 . The black line is the sharp-interface solution. $(b)$ Relative errors for the SBM solutions during diffusion for different $\zeta$ values. Magnified views of $(a)$ at the left $(c)$, and right $(d)$ interfaces.

one must obtain a smooth function, for example, through

$$
\psi=\frac{1}{2}\left[1+\tanh \left(\frac{\varphi}{\zeta}\right)\right],
$$

in which the interfacial thickness is controlled via the value of $\zeta$, and $\varphi$ is the distance function (see appendix E for the detailed procedure). Examples of such calculations are presented in sections 5.1 and 5.3.

Figure 3(a) shows the concentration distributions at $t=1000$ (nearly equilibrium) for $\zeta=2.87 \times 10^{-2}$ (case $\left.1(\mathrm{~b})\right)$ and $\zeta=4.59 \times 10^{-1}$ (case $1(\mathrm{f})$ ), for which $\mathcal{R}_{1}$ values are 83.3 and 5.21, respectively. It is clear that the calculated concentration deviates farther from the sharp-interface solution when the interfacial thickness is larger (with smaller $\mathcal{R}_{1}$ ). Figure $3(b)$ illustrates the relative errors during concentration evolution for various values of $\zeta$. The ratios 
of errors associated with different interfacial thicknesses are roughly constant throughout the evolution, showing transient errors scale similarly with the errors at equilibrium. As the concentration evolution approaches equilibrium, the errors converge to their equilibrium values, as listed in table 1 . The results clearly show that the error increases as the interfacial thickness increases. This agrees with the derivation given in appendix A. A scaling of the error to the interfacial thickness is observed as $\zeta$ is varied from $2.87 \times 10^{-2}$ to $4.59 \times 10^{-1}$ (equivalently an inverse scaling between error and $\mathcal{R}_{1}$ values). In addition, the deviation between the SBM results and the sharp-interface solution is much larger near the left boundary than near the right boundary, indicating the error associated with a Dirichlet boundary condition is larger than that with a Neumann boundary condition; see figures $3(c)-(d)$.

In case 2, we examined the effect of interfacial thickness without changing the number of grid points across the interface; namely, we varied $\mathcal{R}_{1}$ while fixing $\mathcal{R}_{2}$. This was accomplished by selecting various values of $\Delta x$ while maintaining the ratio of interfacial thickness to the grid size at 4.80. The same values of $\mathcal{R}_{1}$ as in case 1 were selected in this set of simulations. Results of concentration distribution (see figures $4(a)-(b)$ ), as well as the magnitudes and scaling of error to interfacial thickness (see case 2 in table 1), similar to case 1 were obtained. Error increases with larger interfacial thicknesses (with larger $\Delta x$ in this case). Since the resolution of the interface is fixed (i.e. $\mathcal{R}_{2}$ is fixed), the result indicates that the increased interfacial thickness is the dominant source of error as long as the variation of domain parameter in the interfacial region can be properly resolved. Based on the results of cases 1 and 2 in table 1, we found that the relative error is very small in a 1D calculation. Even when the interfacial thickness is as large as $19.2 \%$ of the domain size (e.g. $\mathcal{R}_{1}=5.21$ ), the error is on the order of $1 \%$. Interestingly, the errors in case 2 are smaller than those in case 1 for a given value of $\mathcal{R}_{1}$ for sets (c) through (f), which can be attributed to the fact that the interfacial thickness is effectively reduced when the resolution is decreased. This point will be revisited in the next case.

In case 3, we examined the effect of the resolution across the interface while maintaining the interfacial thickness by selecting various $\Delta x$ with the value of $\zeta$ fixed at $5.73 \times 10^{-2}$. Namely, we varied $\mathcal{R}_{2}$ while fixing $\mathcal{R}_{1}$. The results show that error decreases when a lower resolution is selected (with larger $\Delta x$ in this case); see case 3 in table 1 and figures $4(c)-(d)$. This can be understood as follows. As we observed earlier, the SBM formulation reduces to the bulk partial differential equation far from the interface, where the gradient of the domain parameter vanishes. In the interfacial region, the bulk term and the boundary term together set the boundary condition, as shown in appendix A. In between, there is a region where the bulk equation is affected by the boundary term, which is small because the gradient is small, but not negligible. When the resolution is sufficiently low, the domain parameter in bulk regions takes the bulk values, thus vanishing the boundary term and increasing the accuracy. As can be observed in figure $4(d)$, the error behavior in such a case is very different from other cases. In the specific example presented here, the discretized interface at the low resolution of $\mathcal{R}_{2}=1.2$ is nearly a Heaviside step function, which yields smaller error than the higher resolution cases. (When the resolution is high enough, the error is only slightly affected by the resolution.) Therefore, in the 1D case, an interface does not need to be fully resolved, and in fact the accuracy can be increased by not doing so. A similar behavior was observed in case 1(a), which exhibits a smaller error than case 2(a). However, we found that numerical instability ensues when the resolution is further reduced. It has been determined that at least one point with an intermediate value between the two bulk values is required in order to achieve numerical stability.

The argument above applies to only 1D simulations. In multi-dimensional calculations, higher resolution is necessary to ensure that there are a sufficient number of grid points across 

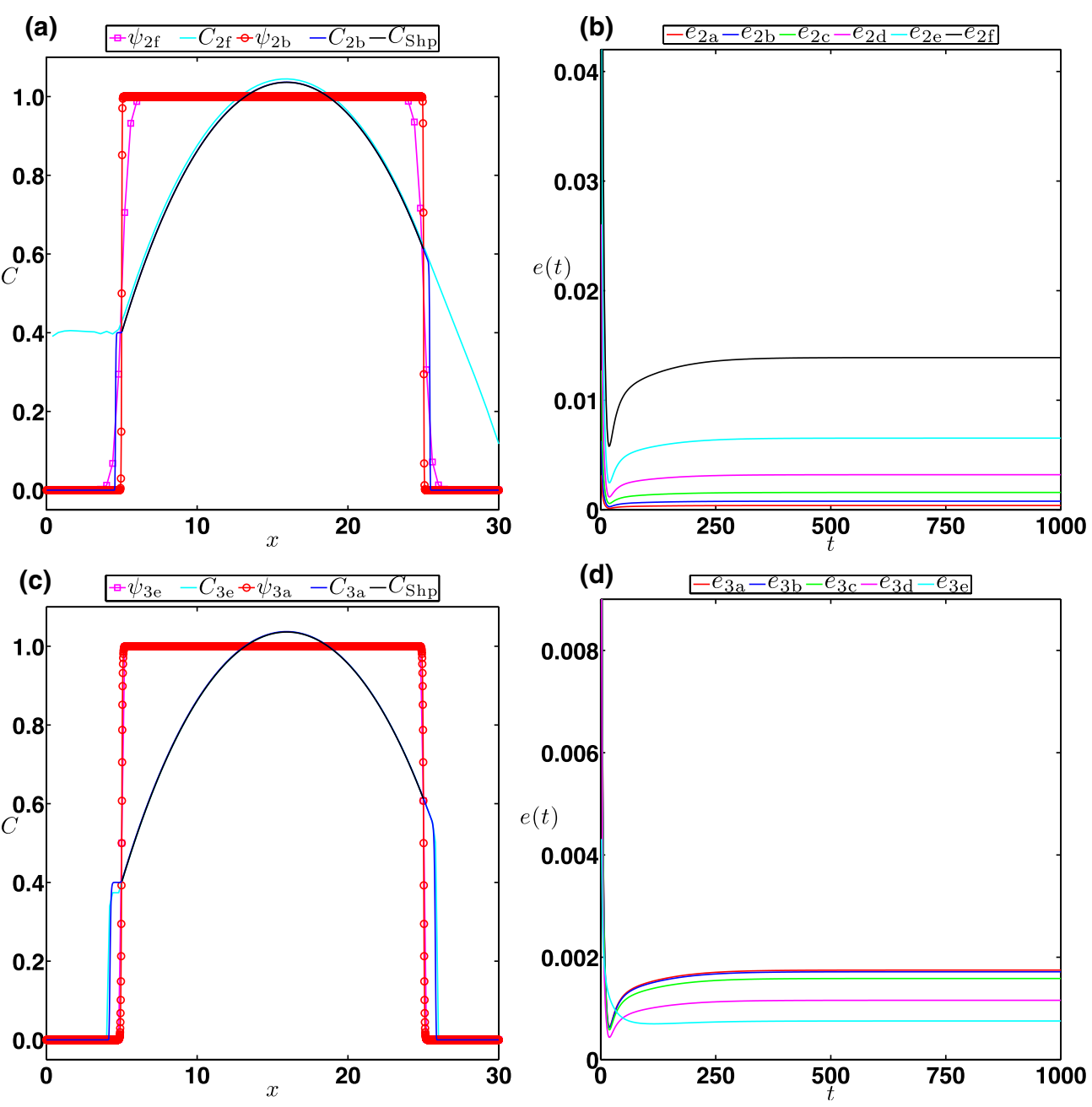

Figure 4. (a) Equilibrium concentrations for cases $2 \mathrm{f}$ and $2 \mathrm{~b}$ in table 1. The black line is the sharpinterface solution. (b) Relative errors for the SBM solutions during diffusion for the parameters in case 2 in table 1. (c) Equilibrium concentrations for cases 3(e) and 3(a). (d) Relative errors for case 3 in table 1.

the interface in all directions of the coordinate system so that interfaces are smooth. This is because a step change in the domain parameter introduces roughness in the interface at the scale of the grid resolution. Therefore, unless the interfaces are flat and coincident with the grid system, it must be smoothed over several grid points.

In case 4 , we varied the value of $v$ (the small value added to the denominators to avoid division by zero) from $1 \times 10^{-2}$ to $1 \times 10^{-11}$, while maintaining $\mathcal{R}_{1}=83.3$ and $\mathcal{R}_{2}=4.80$ by fixing the grid size and interfacial thickness at $\Delta x=2.5 \times 10^{-2}$ and $\xi_{0}=4.8 \Delta x$, respectively. In practice, a smaller $v$ would lead to a less stable numerical implementation because the values of $1 / \psi$ or $1 / \psi^{2}$ become much larger, which requires a much smaller time step size. The results show that the error quickly converges to a small value when $v$ is smaller than $1 \times 10^{-5}$; see case 4 in table 1 . This suggests that once $v$ is small enough to yield converged results, further reduction is unnecessary and should be avoided so that a larger time step can be employed. 
In summarizing the above 1D test simulations, we found that the interfacial thickness (specifically, value of $\mathcal{R}_{1}$ ) is the dominant source of error. The errors are less sensitive to the resolution across the interface (selection of $\mathcal{R}_{2}$ ) and the parameter for singularity control (selection of $v$ ). When the diffuse interface is properly resolved, the error scales with the interfacial thickness. Moreover, in general, the error that results when a Dirichlet boundary condition is imposed is larger and more sensitive to the interfacial thickness than when a Neumann boundary condition is imposed. This behavior can be understood from the results of analysis in appendix A, where the scaling of the errors can be found in equations (A.2) and (A.5).

\subsection{Surface diffusion and bulk diffusion in a cylinder}

To further demonstrate the validity of the SBM, we applied the method to simulate oxygenvacancy diffusion in a cylinder, for which a cylindrical coordinate grid system was used. We solved the coupled surface-bulk diffusion problem using both the SBM and the original sharp-interface formulations in the same grid system for comparison. For the SBM, we used a hyperbolic tangent function, $\psi(r, z)=\{1-\tanh [(R-r)] / \zeta\} / 2$, of the continuous domain parameter to define a cylinder, where $r$ is the radial position, $z$ is the axial position, and $R$ is the cylinder radius. Therefore, the cylinder surface $(\psi=0.5)$, where surface reaction and surface diffusion occur, is located at $r=R$, the solid region $(\psi=1)$ for bulk diffusion is defined at $r<R$ and the environment $(\psi=0)$ is defined at $r>R$. We selected the cylinder radius $R$ and the cylinder axial length to be 1 and 12, respectively. The grid sizes were selected to be $\Delta r=1.76 \times 10^{-2}$ and $\Delta z=4 \times 10^{-2}$, such that the cylinder contains 57 and 300 grid points in the radial and axial directions, respectively. (The computational box is larger than the cylinder in the radial direction, and contains 75 and 300 grid points in the radial and axial directions, respectively.) The interfacial thickness was selected to be $\xi_{0}=4.26 \Delta r$ by setting $\zeta=1.0182 \Delta r$. Equation (12) was solved using a standard central finite difference scheme in cylindrical coordinate system and an Euler explicit time scheme; see appendix B for the discretization scheme. The parameters for the diffusion equation were selected to be $D_{\mathrm{b}}=1$, $D_{\mathrm{s}}=10$ and $L=0$. The boundary conditions at the computation box boundary were set at $C=1$ at $z=0$ and $C=0$ at $z=12$, with no-gradient boundary conditions on the remaining two sides. For comparison, the original sharp-interface equations, (9) and (10), were solved using the same discretization scheme with the same grid system and resolution. For this case, the surface-reaction-diffusion boundary condition, equation (9), was explicitly imposed at the 57th grid points in the radial direction. The concentration evolution is implemented as follows. First, the surface concentration is updated by equation (9) according to the normal flux at the cylinder surface calculated from the normal gradient of the surface concentration obtained from equation (10). Next, the normal surface flux is calculated using equation (9) with the updated surface concentration. The cylinder concentration is then evolved according to equation (10) with the normal flux boundary condition. This procedure is repeated within the Euler explicit time scheme for the concentration evolution. For the SBM formulation, we simply solve a single equation that automatically includes coupled bulk and surface diffusion, equation (12).

Figures $5(a)$ and $(b)$ show the steady-state concentration profiles of the sharp-interface version for $\kappa=2.1$ and $\kappa=50$, respectively. The concentration decays along the axial direction according to boundary values prescribed at the box boundaries. The diffusion front bends because of the surface reaction, such that the concentration is lower near the cylinder surface. Shown in figures $5(c)$ and $(d)$ are the corresponding SBM results. For clarity, only the concentration in the region of $0<z<6 R$ is presented. The results from the two 


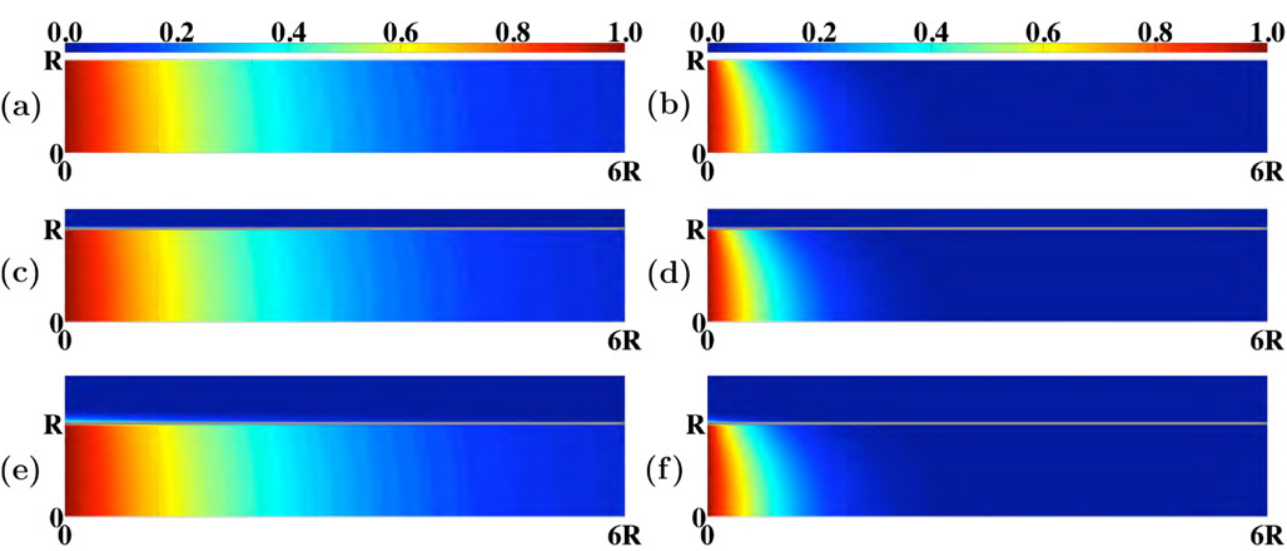

Figure 5. Steady-state concentration profiles for $D_{\mathrm{b}}=1$ and $D_{\mathrm{s}}=10$. The left column is for $\kappa=2.1$, and the right column is for $\kappa=50$. The top row shows the sharp-interface solution; the middle row is the thin-interface SBM results; and the bottom row is the thick-interface SBM results with interfacial thickness four times larger than those in the middle row. The top regions of constant blue color in $(c)-(f)$ represent the areas outside of the solid, whereas the solid white lines indicate the solid cylinder surface.

(a)

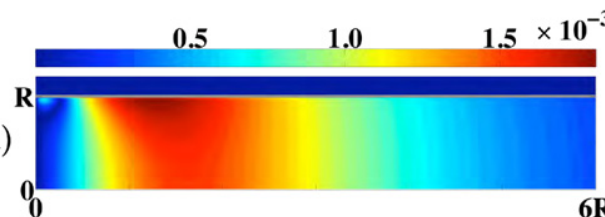

6R

(b)

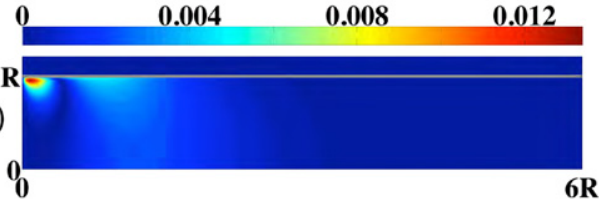

(c)
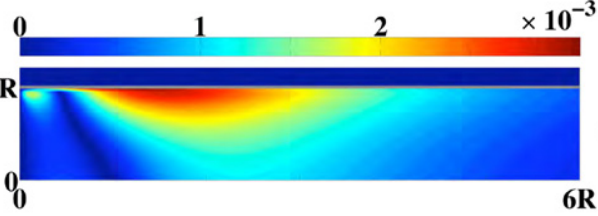

(d)

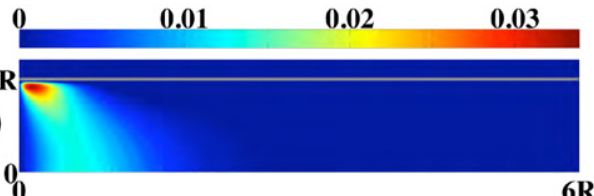

2

(e)

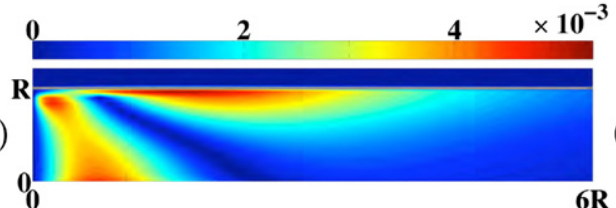

6R

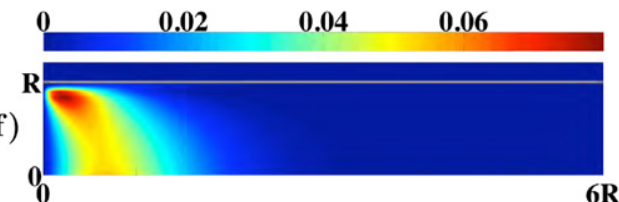

Figure 6. Profiles of relative error between the sharp-interface results and the SBM results having thin, medium and thick interfaces from the top to the bottom: $(a),(c)$ and $(e)$ have $D_{\mathrm{b}}=1, D_{\mathrm{s}}=10$, and $\kappa=2.1$, and are compared with the sharp-interface result shown in figure $5(a) ;(b),(d)$ and $(f)$ have $D_{\mathrm{b}}=1, D_{\mathrm{s}}=10$, and $\kappa=50$, and are compared with the sharp-interface result in figure $5(b)$.

methods are in excellent agreement, clearly demonstrating the utility and validity of the SBM for incorporating two sharp-interface equations into one SBM equation. The relative errors of the results are plotted in figures $6(a)$ and $(b)$. The relative error is calculated by dividing the differences between the SBM and sharp-interface results by the corresponding average concentration of the sharp-interface result. The average concentration is calculated for the active region between the plane at $z=0$ and the plane on which the maximum concentration is 0.01 . Note that only the errors within the cylinder defined by $\psi \geqslant 0.5$ are considered. The 
Table 2. Relative errors for the coupled surface reaction-diffusion and bulk diffusion model in a cylinder, where $e$ denotes the overall error, $e_{\mathrm{b}}$ denotes the bulk error excluding the surface points, and $e_{\mathrm{s}}$ denotes the surface error calculated only with the surface points.

\begin{tabular}{|c|c|c|c|c|c|c|c|c|}
\hline $\begin{array}{l}\zeta=1.0182 \Delta r \\
\mathcal{R}_{2}=4.26\end{array}$ & & $\kappa=2.1$ & & $\kappa=20$ & & $\kappa=50$ & & $\kappa=100$ \\
\hline Thin-interface & $e$ & $7.99 \times 10^{-4}$ & $e$ & $2.26 \times 10^{-3}$ & $e$ & $2.46 \times 10^{-3}$ & $e$ & $7.26 \times 10^{-3}$ \\
\hline$\Delta r=1.76 \times 10^{-2}$ & $e_{\mathrm{b}}$ & $7.99 \times 10^{-4}$ & $e_{\mathrm{b}}$ & $2.23 \times 10^{-3}$ & $e_{\mathrm{b}}$ & $2.39 \times 10^{-3}$ & $e_{\mathrm{b}}$ & $7.37 \times 10^{-3}$ \\
\hline$\xi_{0}=0.075 ; \mathcal{R}_{1}=13.33$ & $e_{\mathrm{S}}$ & $8.03 \times 10^{-4}$ & $e_{\mathrm{S}}$ & $3.02 \times 10^{-3}$ & $e_{\mathrm{S}}$ & $4.02 \times 10^{-3}$ & $e_{\mathrm{S}}$ & $2.63 \times 10^{-3}$ \\
\hline Medium-interface & $e$ & $1.08 \times 10^{-3}$ & $e$ & $3.06 \times 10^{-3}$ & $e$ & $8.32 \times 10^{-3}$ & $e$ & $2.74 \times 10^{-2}$ \\
\hline$\Delta r=3.49 \times 10^{-2}$ & $e_{\mathrm{b}}$ & $1.04 \times 10^{-3}$ & $e_{\mathrm{b}}$ & $2.89 \times 10^{-3}$ & $e_{\mathrm{b}}$ & $8.51 \times 10^{-3}$ & $e_{\mathrm{b}}$ & $2.84 \times 10^{-2}$ \\
\hline$\xi_{0}=0.149 ; \mathcal{R}_{1}=6.71$ & $e_{\mathrm{s}}$ & $1.50 \times 10^{-3}$ & $e_{\mathrm{S}}$ & $4.83 \times 10^{-3}$ & $e_{\mathrm{S}}$ & $5.12 \times 10^{-3}$ & $e_{\mathrm{s}}$ & $1.86 \times 10^{-3}$ \\
\hline Thick-interface & $e$ & $1.81 \times 10^{-3}$ & $e$ & $1.08 \times 10^{-2}$ & $e$ & $2.90 \times 10^{-2}$ & $e$ & $7.34 \times 10^{-2}$ \\
\hline$\Delta r=6.86 \times 10^{-2}$ & $e_{\mathrm{b}}$ & $1.63 \times 10^{-3}$ & $e_{\mathrm{b}}$ & $1.13 \times 10^{-2}$ & $e_{\mathrm{b}}$ & $3.11 \times 10^{-2}$ & $e_{\mathrm{b}}$ & $7.89 \times 10^{-2}$ \\
\hline$\xi_{0}=0.292 ; \mathcal{R}_{1}=3.42$ & $e_{\mathrm{S}}$ & $2.69 \times 10^{-3}$ & $e_{\mathrm{S}}$ & $6.78 \times 10^{-3}$ & $e_{\mathrm{S}}$ & $5.75 \times 10^{-3}$ & $e_{\mathrm{S}}$ & $1.06 \times 10^{-3}$ \\
\hline
\end{tabular}

error values are also tabulated in table 2. The maximum error tends to concentrate at the edge of the cylinder, and is less than $0.2 \%$ and $1.5 \%$ for the two cases considered, respectively.

To examine the effect of the characteristic-length-to-interface ratio (equivalently the interfacial thickness because the system size is fixed), we conducted a set of simulations, in which $\mathcal{R}_{1}$ was varied while fixing $\mathcal{R}_{2}$. Here, the value of $\mathcal{R}_{1}$ is calculated by $\mathcal{R}_{1}=R / \xi_{0}=$ $1 / \xi_{0}$, where the radius of the cylinder (which is smaller than the cylinder length) is taken as the characteristic length. (While the diffusion length is shorter than this in some cases, we do not need to consider it because the SBM is not applied at the base of the cylinder and the diffusion is primarily along the smoothed boundary, rather than perpendicular to it). The diffuse interface is maintained to span $4.26 \Delta r$ (i.e. $\mathcal{R}_{2}=\xi_{0} / \Delta r=4.26$ ). Two additional radial grid sizes, $\Delta r=3.49 \times 10^{-2}$ and $\Delta r=6.86 \times 10^{-2}$, corresponding to numbers of grid points within the cylinders of 29 and 15, respectively, were examined. By selecting the radial grid sizes in this manner, the radial grid points in the thicker-interface case coincide with every other grid point in a thinner-interface case. Hereafter, we refer to the three interfacial thicknesses as the 'thin' ( $\xi_{0}=0.075$ and $\mathcal{R}_{1}=13.33$ ), 'medium' ( $\xi_{0}=0.149$ and $\mathcal{R}_{1}=6.71$ ) and 'thick' ( $\xi_{0}=0.292$ and $\mathcal{R}_{1}=3.42$ ) interface cases. The axial grid size is kept at $\Delta z=4 \times 10^{-2}$. Shown in figures $5(e)$ and $(f)$ are the steady-state concentration profiles for the thick-interface results, corresponding to the cases in figures $5(a)$ and $(b)$. The results are still in reasonably good agreement with the original sharp-interface results, even when the interfacial thickness is approximately $29.2 \%\left(\mathcal{R}_{2}=3.42\right)$ of the cylinder radius.

The relative errors of the thin, medium and thick-interface SBM results are plotted in figure 6. For the thick-interface SBM results the maximum local relative errors for $\kappa=2.1$ and $\kappa=50$ are approximately $5 \times 10^{-3}$ and 0.075 (see figures $6(e)$ and $(f)$ ), whereas the average relative errors are $1.81 \times 10^{-3}$ and $2.90 \times 10^{-3}$, respectively; see table 2 . The average relative errors, denoted by $e$ in table 2, are calculated by dividing the rms deviation between the SBM and the sharp-interface results by the average sharp-interface concentration in the cylinder. The rms deviation and average concentration are calculated in the cylindrical coordinate system. As expected, the error increases as the interface becomes thicker (i.e. as $\mathcal{R}_{1}$ decreases). However, in contrast to the $1 \mathrm{D}$ simple diffusion test in section 4.1 , the behavior of the error is inconsistent across the parameter sets; see table 2. This relatively complicated error behavior may originate from the coupling of the bulk and surface diffusion equations. In addition to the effect of the interfacial thickness, the error also increases with a larger reaction coefficient $\kappa$, which may be explained by the increase in the scaling coefficient for the error 
( $h_{0}$ defined above equation (A.2) in appendix A) when the given boundary value is larger. In addition, the gradient of the concentration near the boundary increases in magnitude with increasing boundary condition value, which can lead to a larger error.

One interesting phenomenon is observed in the high $\kappa$ results $(\kappa=50$ and 100). Although the errors in the bulk substantially increase with the interfacial thickness, the errors at the surface remain small; see figure $6(f)$ and table 2. This indicates that the error originates from the boundary condition affecting the bulk solution, rather than from an increased error in the boundary condition value. The thicker interface thus leads to a larger bulk region that is affected by the boundary condition. Therefore, we compare the error associated with the bulk region and with the boundary condition. Here, the bulk errors, denoted by $e_{\mathrm{b}}$ in table 2 , are calculated by the same method as the average relative error but exclude the grid points on the nominal cylinder surface $(r=R)$. The surface errors, denoted by $e_{\mathrm{s}}$ in table 2 , are calculated by the same method but with only the grid points at the nominal cylinder surface. In the case where $\kappa=100$, the error at the surface even decreases with interfacial thickness.

\subsection{Contact-angle boundary condition}

We conducted simple 2D simulations to validate the SBM formulation for the contact-angle boundary condition at the three-phase boundary. Equations (22) and (23) were tested for nonconserved and conserved order parameters, respectively. The equations were solved using the central finite difference scheme and the Euler explicit time scheme. The computational box sizes are $L_{x}=100$ and $L_{y}=100$, and the parameters used are $\Delta x=1$ and $M=1$. A simple common double-well function was selected for the bulk free energy functional, $f(\phi)=w \phi^{2}(1-\phi)^{2}$, such that the steady-state phase-field order parameter profile is determined by $\phi=\{1-\tanh [(\sqrt{w} \boldsymbol{x}) /(\sqrt{2} \epsilon)]\} / 2$, where $\boldsymbol{x}$ is the coordinate variable indicating the distance to the phase interface, and the thickness of the diffuse phase interface is given by $\xi_{\phi}=4.185 \delta_{\phi}$, where $\delta_{\phi}=\epsilon \sqrt{2 / w}$. We control the phase-interface thickness while fixing the interfacial energy by setting $\epsilon=\sqrt{1 / w}$, which leads to $\delta_{\phi}=\sqrt{2} / w$ and $\gamma_{\phi}=\epsilon \sqrt{2 w} / 6=\sqrt{2} / 6$.

In this set of simulations, we have two diffuse interfaces: one for the phase-field order parameter and the other for the SBM domain parameter. A horizontal diffuse domain interface representing a flat substrate surface is defined by the hyperbolic tangent function $\psi=\{1+\tanh [(y-30) / \zeta]\} / 2$, such that $\psi=0.5$ is at $y=30$, and $\psi$ gradually transitions from 0 to 1 from below to above the substrate surface. An initial phase interface was placed vertically in the middle of the domain $(x=50)$, with phase $1(\phi=1)$ and phase $0(\phi=0)$ on the left and right halves, respectively. On the computational box boundaries, the normal gradients of the phase-field order parameter were set at zero: $\partial \phi / \partial x=0$ at $x=0$ and 100, and $\partial \phi / \partial y=0$ at $y=0$ and 100, which can be interpreted as the no-flux boundary conditions.

There are two values that could serve as the characteristic length here for determining $\mathcal{R}_{1}$. One is the domain size along the $y$-axis because the domain interface is flat, and its value is $L_{y}^{\Omega}=70$ measured between the domain interface and the top of the computational box. The other is the radius of the curvature of the phase interface, whose value can be calculated according to $R_{\phi}=70 /\left|\cos \theta_{\mathrm{B}}\right|$ in these simulations because a $90^{\circ}$ intersection between the phase interface and the top box boundary is formed due to the no-flux boundary condition imposed at the top box boundary. Since $R_{\phi}$ is always larger than or equal to $L_{y}^{\Omega}$, we take $L_{\mathrm{c}}=L_{y}^{\Omega}=70$ as the characteristic length, and thus $\mathcal{R}_{1}=70 / \xi_{0}$. In addition, the characteristic length can be compared with the phase-interface thickness to obtain another ratio: $\mathcal{R}_{3}=70 / \xi_{\phi}$. Both ratios can affect the accuracy when imposing contact-angle boundary conditions. Therefore, various combinations of the two ratios were examined to evaluate how 

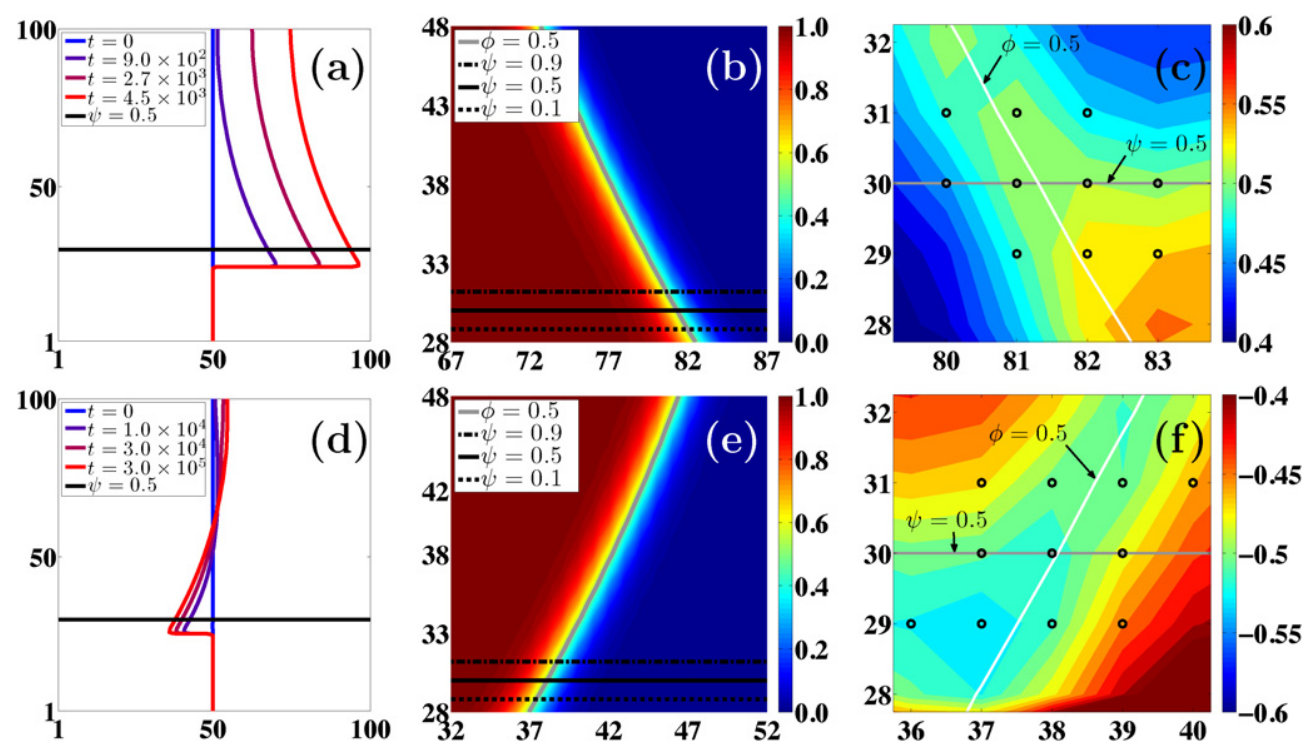

Figure 7. (a) Contour lines of $\psi=0.5$ at various times for Allen-Cahn-type evolution equation with a $60^{\circ}$ contact-angle boundary condition. $(b)$ Magnified view of the order parameter and $(c)$ $\cos \theta$ profiles near the three-phase boundary (further magnified), corresponding to $t=2.7 \times 10^{3}$ in $(a)$. (d) Contour lines of $\psi=0.5$ at various times for Cahn-Hilliard-type evolution equation with a $120^{\circ}$ contact-angle boundary condition. (e) Magnified view of the order parameter and (f) $\cos \theta$ profiles at the three-phase boundary (further magnified), corresponding to $t=3.0 \times 10^{5}$ in $(d)$. The cosine values of the imposed contact angles are 0.5 and -0.5 for $(a)-(c)$ and $(d)-(f)$, respectively. The open circles in $(c)$ and $(f)$ denote the grid points in the range $0.1<\psi<0.9$ and $0.1<\phi<0.9$. The order parameters in the region of $\psi<0.5$ have no physical significance. For the Cahn-Hilliard case, the order parameter is conserved in the region of $\psi>0.5$.

the accuracy of the SBM depends on these parameters when imposing contact-angle boundary conditions.

In the first set of simulations, we evolved equation (22) for a nonconserved order parameter with a $60^{\circ}$ contact angle. The result clearly shows a $60^{\circ}$ contact angle at the three-phase boundary, as specified; see figure 7(a). The angle can be measured at the intersection between the two contours of $\psi=0.5$ and $\phi=0.5$, as shown in figures $7(b)$ and $(c)$. Note that the order parameter takes a nonzero value below the nominal substrate surface because the substrate surface is defined by a gradually varying domain parameter and thus having a nonzero thickness. In the simulation, the region of order parameter evolution extends to near the contour line where $\psi \sim 1.0 \times 10^{-5}$ (the small nonzero value added to the denominator in equation (22)). The $60^{\circ}$ angle is maintained during the entire evolution, except for the very early transient period, when the contact angle changes from the initial $90^{\circ}$ angle to the prescribed $60^{\circ}$ angle. Because of the contact-angle boundary condition, the initially flat phase interface bends and creates a negative curvature in phase 1 . As a result, the phase interface moves toward phase 0 . Once the phase interface evolves to a circular arc with a uniform curvature everywhere (other than regions in contact with the domain interface), it moves at a uniform constant speed in a steady-state motion, and eventually only phase 1 remains in the system.

In the second set of simulations, we evolved equation (23) for a conserved order parameter in a closed system with $J_{n}=0$ and a $120^{\circ}$ contact angle. As expected, the phase interface intersects the domain interface at a $120^{\circ}$ contact angle; see figures $7(d)-(f)$. In contrast to 
Table 3. The errors and cosines of contact angles from the validation simulations using the AllenCahn equation and the Cahn-Hilliard equation. In addition, a measure of the order parameter conservation, evaluated by $\int \psi \phi\left(t_{\mathrm{ss}}\right) \mathrm{d} \Omega / \int \psi \phi(t=0) \mathrm{d} \Omega$, where $t_{\mathrm{ss}}$ is the time required to reach steady-state conditions and $\Omega$ is the computational domain, is presented for simulations with the Cahn-Hilliard equation.

\begin{tabular}{|c|c|c|c|c|c|c|c|}
\hline$\zeta$ & $\xi_{0}$ & $\mathcal{R}_{1}$ & $\begin{array}{l}\delta_{\phi}=1.0607 \\
(w=1.33) \\
\xi_{\phi}=4.44 \\
\mathcal{R}_{3}=15.77\end{array}$ & $\begin{array}{l}\delta_{\phi}=1.4142 \\
(w=1.00) \\
\xi_{\phi}=5.92 \\
\mathcal{R}_{3}=11.82\end{array}$ & $\begin{array}{l}\delta_{\phi}=1.7678 \\
(w=0.80) \\
\xi_{\phi}=7.40 \\
\mathcal{R}_{3}=9.46\end{array}$ & $\begin{array}{l}\delta_{\phi}=2.1213 \\
(w=0.67) \\
\xi_{\phi}=8.88 \\
\mathcal{R}_{3}=7.88\end{array}$ & $\begin{array}{l}\delta_{\phi}=2.8284 \\
(w=0.50) \\
\xi_{\phi}=11.84 \\
\mathcal{R}_{3}=5.91\end{array}$ \\
\hline & & & \multicolumn{5}{|c|}{ Error and cosine of contact angle for Allen-Cahn equation } \\
\hline 0.75 & 3.14 & 22.22 & $2.50 \mathrm{E}-3(0.5050)$ & $2.40 \mathrm{E}-3(0.5048)$ & $1.75 \mathrm{E}-3(0.4965)$ & $5.00 \mathrm{E}-5(0.5001)$ & $2.00 \mathrm{E}-4(0.5004)$ \\
\hline 1.00 & 4.19 & 16.67 & $5.00 \mathrm{E}-3(0.4900)$ & $1.95 \mathrm{E}-3(0.5039)$ & $1.70 \mathrm{E}-3(0.4966)$ & $9.00 \mathrm{E}-4(0.4982)$ & $2.20 \mathrm{E}-3(0.4956)$ \\
\hline 1.50 & 6.28 & 11.11 & $6.75 \mathrm{E}-3(0.4865)$ & $1.90 \mathrm{E}-3(0.4962)$ & $3.65 \mathrm{E}-3(0.4927)$ & $3.10 \mathrm{E}-3(0.4938)$ & $3.65 \mathrm{E}-3(0.4927)$ \\
\hline 2.00 & 8.37 & 8.33 & $5.70 \mathrm{E}-3(0.4886)$ & $4.10 \mathrm{E}-3(0.4918)$ & $5.85 \mathrm{E}-3(0.4883)$ & $4.95 \mathrm{E}-3(0.4901)$ & $4.95 \mathrm{E}-3(0.4901)$ \\
\hline 4.00 & 16.7 & 4.17 & $8.75 \mathrm{E}-3(0.4825)$ & $1.09 \mathrm{E}-2(0.4782)$ & $1.09 \mathrm{E}-2(0.4783)$ & $1.03 \mathrm{E}-2(0.4795)$ & $1.05 \mathrm{E}-2(0.4790)$ \\
\hline \multicolumn{8}{|c|}{ Error and cosine of contact angle for Cahn-Hilliard equation } \\
\hline 0.75 & 3.14 & 22.22 & $8.45 \mathrm{E}-3(-0.4831)$ & $1.50 \mathrm{E}-4(-0.5003)$ & $3.15 \mathrm{E}-3(-0.4937)$ & $1.05 \mathrm{E}-3(-0.4979)$ & $3.45 \mathrm{E}-3(-0.4931)$ \\
\hline 1.00 & 4.19 & 16.67 & $3.85 \mathrm{E}-3(-0.4923)$ & $3.70 \mathrm{E}-3(-0.4926)$ & $5.15 \mathrm{E}-3(-0.4897)$ & $3.55 \mathrm{E}-3(-0.4929)$ & $5.95 \mathrm{E}-3(-0.4881)$ \\
\hline 1.50 & 6.28 & 11.11 & $7.95 \mathrm{E}-3(-0.4841)$ & $6.45 \mathrm{E}-3(-0.4871)$ & $6.60 \mathrm{E}-3(-0.4868)$ & $5.50 \mathrm{E}-3(-0.4890)$ & $6.65 \mathrm{E}-3(-0.4867)$ \\
\hline 2.00 & 8.37 & 8.33 & $1.81 \mathrm{E}-2(-0.4639)$ & $7.15 \mathrm{E}-3(-0.4857)$ & $6.95 \mathrm{E}-3(-0.4861)$ & $6.90 \mathrm{E}-3(-0.4862)$ & $7.35 \mathrm{E}-3(-0.4853)$ \\
\hline 4.00 & 16.7 & 4.17 & $3.14 \mathrm{E}-2(-0.4372)$ & $1.43 \mathrm{E}-2(-0.4713)$ & $1.24 \mathrm{E}-2(-0.4752)$ & $1.28 \mathrm{E}-2(-0.4745)$ & $1.24 \mathrm{E}-2(-0.4752)$ \\
\hline \multicolumn{8}{|c|}{ Conservation of order parameter for Cahn-Hilliard equation } \\
\hline 0.75 & 3.14 & 22.22 & 0.9929 & 0.9972 & 0.9979 & 0.9982 & 0.9986 \\
\hline 1.00 & 4.19 & 16.67 & 0.9930 & 0.9973 & 0.9979 & 0.9982 & 0.9986 \\
\hline 1.50 & 6.28 & 11.11 & 0.9933 & 0.9974 & 0.9980 & 0.9983 & 0.9987 \\
\hline 2.00 & 8.37 & 8.33 & 0.9976 & 0.9991 & 0.9993 & 0.9994 & 0.9996 \\
\hline 4.00 & 16.7 & 4.17 & 0.9982 & 0.9993 & 0.9995 & 0.9996 & 0.9997 \\
\hline
\end{tabular}

the Allen-Cahn-type dynamics, because of the conservation of the order parameter, the phase interface near the substrate surface moves toward the left, whereas the phase interface away from the substrate moves in the opposite direction. As a result, the phase interface deforms into a curved shape. When the system reaches its equilibrium state, the phase interface forms a circular arc with a uniform curvature everywhere (except where the phase is in contact with the substrate), such that the total surface energy is minimized; see figure $7(d)$ for $t=3.0 \times 10^{5}$.

Table 3 lists the calculated cosine values of the contact angle at the three-phase boundary and their errors. The estimated $\cos \theta$ was calculated by averaging the values of $(\nabla \psi \cdot \nabla \phi) /(|\nabla \psi||\nabla \phi|)$ at the grid points within the three-phase boundary region defined by $0.1<\psi<0.9$ and $0.1<\phi<0.9$ in a steady state. The error was estimated via $\left(\cos \theta-\cos \theta_{\mathrm{B}}\right) / 2$, where the factor of two in the denominator stems from the total variation of the magnitude of the cosine function. Since there were at least 3.14 grid points spanning the domain interface in these simulations, the resolution over a flat domain interface should be sufficient for numerical accuracy. According to section 4.1, the error mainly originate from $\mathcal{R}_{1}$, and is less sensitive to $\mathcal{R}_{2}$. Therefore, we only varied the values of $\zeta$ with fixed $\Delta x$ in this parametric study to examine the effect of $\mathcal{R}_{1}$. These results again clearly show that the error (for a given phase-interface thickness) increases as the domain-interface thickness increases and are consistent with the 1D results.

In contrast to the effect of domain-interface thickness, the error is relatively insensitive to the phase-interface thickness once the phase interfaces are properly resolved; see cases with $\delta_{\phi} \geqslant 1.4142$ in table 3 . However, when the phase interface is very thin, the error tends to increase because of the insufficient resolution of the phase interface (4.44 grid spacings between order parameter values of 0.015 to 0.985 ); see cases with $\delta_{\phi}=1.0607$ in table 3 . 
However, in general, the results demonstrate that the contact-angle boundary condition is well imposed using the SBM. Even when the domain-interface thickness is as large as $\xi_{0}=16.74$ $\left(\mathcal{R}_{1}=4.17\right.$ ), the error in $\cos \theta$ is around $1 \%$ (corresponding to an error of about $2^{\circ}$ in the contact angle) as long as the phase interface is properly resolved.

In addition to the contact angle, a no-flux boundary condition for a conserved order parameter is imposed at the domain interface. The error associated with such a boundary condition was evaluated by examining the overall change in the value of the total order parameter. The conservation of the order parameter was met within a numerical error (well below $1 \%$ in most cases) in these validation simulations; see table 3 .

\section{Applications}

We have so far presented the mathematical derivations and numerical tests of the SBM in sections 3 and 4, respectively. To demonstrate its potential, we now present four example applications in materials science. Brief background information accompanies each of the examples, but it is beyond the scope of this article to provide the complete details. Such details will be published in the future along with research results generated using this method.

\subsection{Oxygen-vacancy diffusion in an SOFC cathode}

An SOFC contains three main components: cathode, anode and electrolyte. The cathode typically consists of interwoven pore channels and ceramic particles, which provide transport paths for air and oxygen ions, respectively. The anode typically consists of interconnected metal particles for electron conduction, pore channels for hydrogen/hydrocarbon fuel delivery, and interconnected ceramic particles for oxygen ion transport. The electrolyte is a solid ceramic layer separating the cathode and anode, allowing only oxygen ion transport. At the cathode particle surfaces, oxygen gas molecules react with electrons to form oxygen ions. These ions diffuse along the surfaces and through the bulk of the cathode particles and the electrolyte, and then finally diffuse through ceramic anode particles to react with fuel molecules. Because the ceramic materials in the electrodes and electrolyte have well-defined stoichiometry, oxygen ion diffusion is equivalent to vacancy diffusion within the oxygen sublattice of the oxide in the opposite direction, where the vacancies at oxygen sub-lattice sites are referred to as oxygen vacancies. To simulate oxygen-vacancy diffusion in a complex SOFC microstructure, equation (12) was solved with the continuous domain parameter, $\psi$, defining the geometry of the microstructure. We adopted an experimentally reconstructed complex microstructure comprised of a porous ceramic cathode and a nonporous ceramic electrolyte of an SOFC, as the input geometry. The microstructure data are stored as a 3D array consisting of $321 \times 261 \times 297$ voxels that indicate the electrolyte (gadolinium-doped ceria: GDC), cathode (lanthanum strontium chromite: LSC) and pore phases by different values. To emphasize the convenience of image-based SBM simulations, we treat the center of each voxel as the location of the grid points in the calculation without further enhancement of the resolution from our initial reconstructed microstructure. For very high-accuracy scientific calculations, one can easily enhance the resolution by refining the grid sizes. To smooth the voxelated, discontinuous data, we first employed a level set distance function method [44] to determine the distances between grid points and the solid-pore interface, and then computed the hyperbolic tangent (equation (27)) of the distance function to obtain the domain parameter profile; see appendix E for details.

For simulations of the concentration distribution in the porous cathode, the regions containing nonporous electrolyte are excluded, such that the computational box only consists 

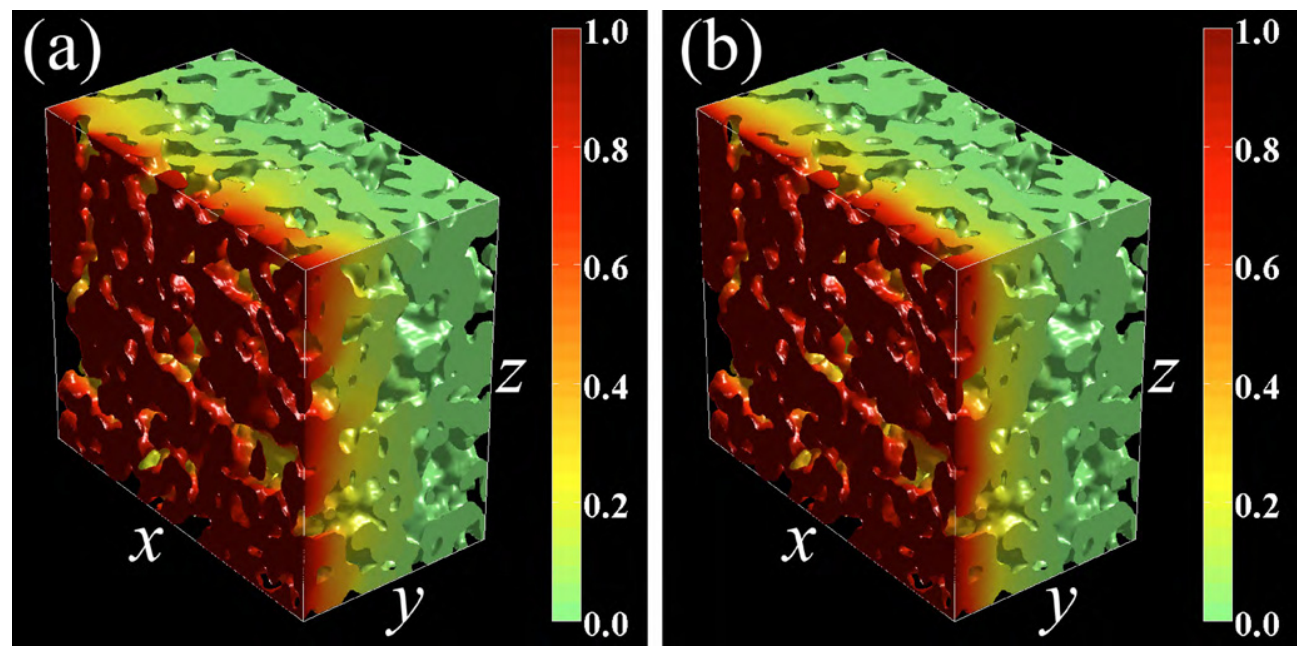

Figure 8. Simulation results of dimensionless steady-state oxygen-vacancy concentrations for $D_{\mathrm{b}}=1$ using an experimentally obtained SOFC cathode complex microstructure as the input geometry: $(a) \kappa=0.1$ and $D_{\mathrm{s}}=0 ;(b) \kappa=2.1$ and $D_{\mathrm{s}}=10$.

of $321 \times 176 \times 297$ grid points. The spatial dimension is normalized to a characteristic cylindrical cathode-particle radius given by $R_{\mathrm{c}}=2 / S_{\mathrm{v}}=0.398 \mu \mathrm{m}$, where $S_{\mathrm{v}}=5.03 \mu \mathrm{m}^{-1}$ is the surface area per unit volume of the microstructure. The factor of two arises as we estimate the feature size of the complex microstructure from the surface area per unit volume of an array of cylinders. The dimensionless grid spacing is set at $\Delta x=6.285 \times 10^{-2}$ and the interfacial thickness is set by $\zeta=0.051$ in equation (27), such that $\mathcal{R}_{1}=4.69$ and $\mathcal{R}_{2}=3.39$. This set of parameters may result in errors between those given for the medium-interface and thick-interface cases in section 4.2 and table 2, which are well below $1 \%$ for the $\kappa$ value examined below. The boundary conditions on the computational box at $y=0$ and $y=11.062$ are $C=1$ and $C=0$, respectively, and zero-gradient on the remaining four sides. The input parameters to the dimensionless governing equation were taken from [42]. Figure 8 shows the steady-state concentrations for the cases in which surface diffusion is excluded $(\kappa=0.1$ and $\left.D_{\mathrm{s}}=0\right)$ and included $\left(\kappa=2.1\right.$ and $\left.D_{\mathrm{s}}=10\right)$ with $D_{\mathrm{b}}=1$ for both cases. The concentration decays from 1 to 0 along the $y$-axis over the complex cathode microstructure to satisfy the boundary conditions imposed on the box boundaries and at the cathode-pore interfaces. The utilization lengths (i.e. the length over which the cathode material is active) of the two cases are similar, as predicted by Lu et al [42] for a cathode with simplified cylindrical geometry, in which the effective diffusivities for the two parameter sets given above are found to be similar. However, a slight difference in the concentration distributions of the two cases can be observed. Because of the faster transport path along the surface, the diffusion front with surface diffusion (figure $8(b)$ ) is more planar compared with the case without surface diffusion (figure 8(a)).

The SBM can also be used to impose Dirichlet boundary conditions on irregular surfaces. For example, if the ionic diffusivity in the electrolyte is assumed to be much larger than that in the cathode, the concentration in the electrolyte will be nearly uniform. To simulate this scenario, we impose a fixed concentration at the electrolyte-cathode contacting surface as the boundary condition. We used the domain parameter profiles as our input geometry that was smoothed from the experimentally reconstructed $321 \times 261 \times 297$ voxel array containing a 

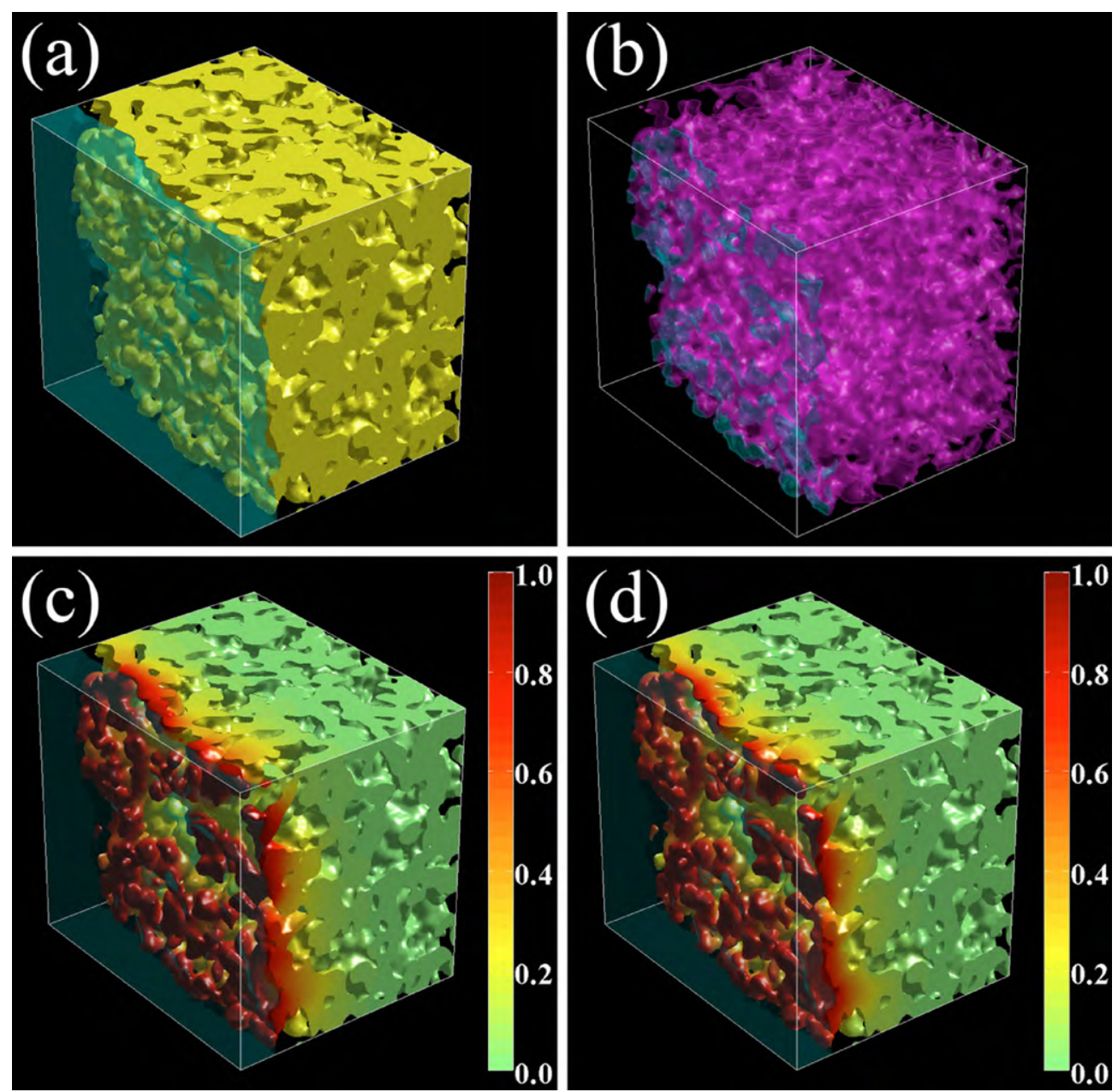

Figure 9. (a) Solid phase containing cathode (yellow) and electrolyte (semitransparent cyan) in a solid oxide fuel cell material. (b) Interfaces defined by $\sqrt{\left(\left|\nabla \psi_{1}\right|\left|\nabla \psi_{2}\right|\right)}>0.2$ (semitransparent cyan) and $\sqrt{\left(\left|\nabla \psi_{2}\right|\left|\nabla \psi_{3}\right|\right)}>0.2$ (semitransparent purple). Dimensionless steady-state oxygenvacancy concentration with the boundary condition $C=1$ at the electrolyte-cathode interface for $D_{\mathrm{b}}=1:(c) \kappa=0.1$, and $D_{\mathrm{s}}=0 ;(d) \kappa=2.1$, and $D_{\mathrm{s}}=10$.

porous cathode and a nonporous electrolyte. Here, three domain parameters are employed to define the three regions: electrolyte $\left(\psi_{1}\right)$, cathode $\left(\psi_{2}\right)$ and pore $\left(\psi_{3}=1-\psi_{1}-\psi_{2}\right)$. The SBM formulated governing equation is obtained by modifying equation (12) to

$$
\begin{array}{r}
\frac{\partial C}{\partial t}=\frac{\nabla \cdot\left(\psi_{2} D_{\mathrm{b}} \nabla C\right)}{\psi_{2}}-\frac{\left|\nabla \psi_{2}\right|}{\psi_{2}}\left[\kappa C-D_{\mathrm{s}} \nabla_{\mathrm{s}}^{2} C\right] W_{\mathrm{N}} \\
-\frac{D_{\mathrm{b}}}{\psi_{2}^{2}}\left[\nabla \psi_{2} \cdot \nabla\left(\psi_{2} C\right)-\left|\nabla \psi_{2}\right|^{2} B_{\mathrm{D}}\right] W_{\mathrm{D}},
\end{array}
$$

where the weighting factors are given by $W_{\mathrm{N}}=\left[\left|\nabla \psi_{2}\right|\left|\nabla \psi_{3}\right| /\left(\left|\nabla \psi_{1}\right|\left|\nabla \psi_{2}\right|+\left|\nabla \psi_{2}\right|\left|\nabla \psi_{3}\right|+\right.\right.$ $\left.\left.\left|\nabla \psi_{3}\right|\left|\nabla \psi_{1}\right|\right)\right]^{\beta}$ and $W_{\mathrm{D}}=\left[\left|\nabla \psi_{1}\right|\left|\nabla \psi_{2}\right| /\left(\left|\nabla \psi_{1}\right|\left|\nabla \psi_{2}\right|+\left|\nabla \psi_{2}\right|\left|\nabla \psi_{3}\right|+\left|\nabla \psi_{3}\right|\left|\nabla \psi_{1}\right|\right)\right]^{\beta}$, such that the Neumann boundary condition (surface reaction and surface diffusion) is imposed only at the cathode-pore interface $\left(\left|\nabla \psi_{2}\right|\left|\nabla \psi_{3}\right| \neq 0\right)$, and the Dirichlet boundary condition 
(a prescribed concentration value) is imposed only at the electrolyte-cathode interface $\left(\left|\nabla \psi_{1}\right|\left|\nabla \psi_{2}\right| \neq 0\right)$. The exponent $\beta$ determines the transition profiles from the Neumann to the Dirichlet boundary conditions in the regions of three-phase boundaries. We selected $\beta=0.8$ for this numerical simulation. On the computational box boundaries, we set $C=0$ at $y=16.404$ and the zero-gradient boundary condition for the remaining five sides.

The same material parameters used in the cases of figure 8 were selected. Figures $9(a)$ and (b) illustrate the reconstructed SOFC complex microstructure and irregular surfaces defined by the values and gradients of the domain parameters, respectively. Figures $9(c)$ and $(d)$ show the simulation results of the steady-state oxygen-vacancy concentration distributions with a fixed value of $C=1$ imposed at the cathode (LSC)-electrolyte (GDC) interfaces. The concentration distribution is very different from the ones shown in figure 8 because a larger portion of lateral diffusion occurs in the $x$ and $z$ directions, which results from the smaller contacting areas (compared with the cross-sectional area of LSC on the $x-z$ plane in figure 8 , where diffusion is mainly in the $y$ direction). As a result, the concentration drops rapidly within a short distance from the contacting areas, making the utilization length of the cathode material shorter and uneven.

\subsection{Kirkendall-effect diffusion and deformation with a moving boundary}

The second application demonstrates the SBM's broad applicability by coupling the SBM formulated diffusion equation derived in section 3.2 to the Navier-Stokes-Cahn-Hilliard equations [45-50] as a model of plastic deformation due to volume expansion and contraction resulting from vacancy flow.

In a binary substitutional alloy, there are three diffusing species: two atomic species and a dilute concentration of vacancies. The two atomic species typically have different mobilities, and thus the two fluxes of the atomic species do not fully compensate for each other. This leads to a net vacancy flux from the slow diffuser side toward the fast diffuser side. In Darken's model [51,52], vacancy sources/sinks are assumed to be efficient and abundant in a solid, and therefore vacancies are immediately generated/eliminated to maintain its concentration at the thermal-equilibrium value everywhere in the diffusion couple. In this case, the solid experiences deformation because of the local expansion and shrinkage [53-57] resulting from vacancy generation and elimination, respectively. This vacancy flux leads to the motion of markers embedded in the diffusion couple and/or deformation of the diffusion couple, known as the Kirkendall effect [51, 52, 58-60]. To model the coupled diffusion-deformation problem, we treat the solid as a very viscous fluid [61-65] with a much larger viscosity than that of the surrounding environment.

We solve the Navier-Stokes-Cahn-Hilliard equations to update the shape of the material as follows [66]:

$$
\begin{aligned}
& -\nabla P+\nabla \cdot \eta\left[\nabla \boldsymbol{v}+(\nabla \boldsymbol{v})^{\mathrm{T}}\right]-\nabla\left(\frac{2 \eta}{d} g_{\mathrm{V}}\right)+\frac{1}{C_{\mathrm{a}}} \mu \nabla \psi=0 \\
& \nabla \cdot \boldsymbol{v}=g_{\mathrm{V}} \\
& \frac{\partial \psi}{\partial t}-\boldsymbol{v} \cdot \nabla \psi=M \nabla^{2}\left(\frac{\partial f}{\partial \psi}-\epsilon^{2} \nabla^{2} \psi\right)
\end{aligned}
$$

where $P$ is the effective pressure, $\eta$ is the viscosity, $v$ is the velocity vector, $d$ is the number of dimensions, the superscript $\mathrm{T}$ denotes the transpose, $C_{\mathrm{a}}$ is the Cahn number reflecting the capillary force compared with the pressure gradient, $g_{\mathrm{V}}$ is the vacancy generation rate per unit volume, and $\psi$ is the domain parameter indicating the solid phase for diffusion. The value 
of viscosity transitions from a small value corresponding to the surrounding medium phase where $\psi=0$ to a large value corresponding to the deformable solid where $\psi=1$. It is convenient to interpolate the viscosity coefficient by the domain parameter as $\eta=\bar{\eta} \psi$, where $\bar{\eta}$ is a constant viscosity coefficient for the solid phase. Equations (29a) and (29b) govern the relationship between the material flow and local volume expansion rate (which is equivalent to vacancy generation rate). Note that the two phases (solid and surrounding vapor) together in the framework of Navier-Stokes-Cahn-Hilliard model [45-50] are treated as a single twophase fluid with very different viscosities in each phase. Therefore, the pressure and velocity fields are continuous across the interface; i.e. a no-slip boundary condition for velocity. One great advantage in employing a phase-field-type equation is that it automatically maintains the profile of the domain parameter, $\psi$, in the form of a hyperbolic tangent function because it is the equilibrium solution for the phase-field equation $(29 c)$. Note that here we ignore the inertial force in the Navier-Stokes equation to obtain equation (29a) because the deformation is assumed to be a quasi-steady-state process. The vacancy generation rate that results in the local volume change (dilatational strain) is given by $g_{\mathrm{V}}=-\left[\nabla \cdot\left(D_{\mathrm{VB}} \nabla X_{\mathrm{B}}\right)\right] /\left[\rho_{1}\left(1-X_{\mathrm{V}}^{\mathrm{eq}}\right)\right]$, where $X_{\mathrm{B}}$ is the mole fraction of the fast diffuser, $X_{\mathrm{V}}^{\mathrm{eq}}$ is the thermal-equilibrium vacancy mole fraction (which is assumed to be maintained throughout the solid in this model), $D_{\mathrm{VB}}$ is the diffusivity for vacancy flux associated with $\nabla X_{\mathrm{B}}$, and $\rho_{\mathrm{l}}$ is the lattice site density of the solid. The evolution of the fast diffuser mole fraction is governed by the advective Fick's diffusion equation, written as:

$$
\frac{\partial X_{\mathrm{B}}}{\partial t}-\boldsymbol{v} \cdot \nabla X_{\mathrm{B}}=\nabla \cdot\left(D_{\mathrm{BB}}^{\mathrm{V}} \nabla X_{\mathrm{B}}\right)-X_{\mathrm{B}} g_{\mathrm{V}},
$$

where $D_{\mathrm{BB}}^{\mathrm{V}}$ is the diffusivity for the fast diffuser flux associated with $\nabla X_{\mathrm{B}}$, and the advective term accounts for the lattice shift because of volume change. Because diffusing atoms cannot depart from the solid region, a no-flux boundary condition is imposed at the solid surface. Thus, the SBM formulation of equation (30) is written as

$$
\frac{\partial X_{\mathrm{B}}}{\partial t}-\boldsymbol{v} \cdot \nabla X_{\mathrm{B}}=\frac{\nabla \cdot\left(\psi D_{\mathrm{BB}}^{\mathrm{V}} \nabla X_{\mathrm{B}}\right)}{\psi}-X_{\mathrm{B}} g_{\mathrm{V}} \cdot
$$

As the concentration evolves, the shape of the solid is also updated by equation $(29 c)$ and by iteratively solving equations $(29 a)$ and $(29 b)$ through the application of a projection method [67, 68]; see appendix F for the numerical implementation.

For this simulation, we use the theoretically calculated diffusivities [69-72] that are available for thermodynamically ideal random alloys. We use the material properties calculated for aluminum at $600 \mathrm{~K}$ for the slow diffuser, and the fast diffuser is assumed to have a smaller migration barrier such that the success rate of atomic hops is four times larger than the slow diffuser. The thermal-equilibrium vacancy mole fraction, $X_{\mathrm{V}}^{\mathrm{eq}}$, is taken to be $1.6 \times 10^{-6}$. The slow and fast diffusers are initially placed in the left and right halves of the solid, respectively. A rectangular diffusion couple spanning $192 \times 64$ grid points is immersed in a computational box containing $256 \times 128$ grid points. The grid spacing is used to nondimensionalize the length scale, and thus $\Delta x=1$. Here, since we are treating a solid as a highly viscous fluid, the capillary force should be negligible compared with the pressure across the solid surface. Thus, we set $C_{\mathrm{a}}=1 \times 10^{8}$. In addition, the deformation must be driven by the advection, rather than the phase transformation, since the latter is solely used as a numerical tool for tracking the solid surface. Therefore, we set $M=1.25 \times 10^{-8}$. Other parameters are given by $\bar{\eta}=1 \times 10^{5}$ (providing a large viscosity for the solid) and $\epsilon=1$ (providing diffuse interface that can be resolved numerically). The same double-well free energy function with $w=1$ as in section 4.3 is used in equation (29c), such that the interfacial thickness is $\xi_{0}=5.92$; thus, $\mathcal{R}_{1}$ is approximately 16.2 in the primary diffusion direction, and $\mathcal{R}_{2}=5.92$. Based on the study 

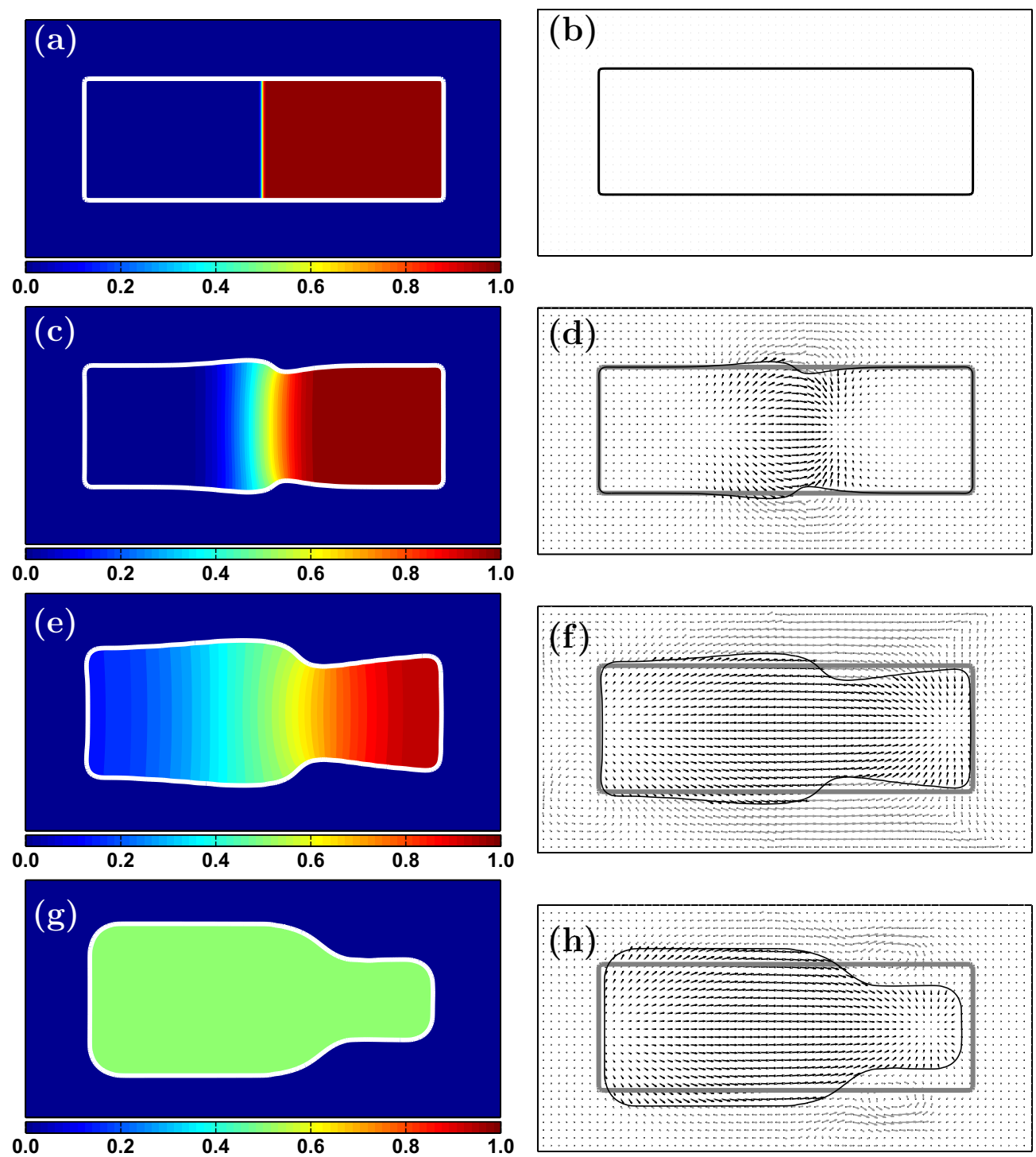

Figure 10. Left column: fast diffuser mole fraction profiles (normalized to the lattice site density) recorded at dimensionless time of $t=0,3.96 \times 10^{7}, 5.09 \times 10^{8}$ and $4.32 \times 10^{9}$. Right column: velocity fields corresponding to the mole fraction profile on the left. Black and gray arrows denote the flows inside and outside the material, respectively. The flow outside of the material has no physical significance for the shape change. Simulation parameters were $C_{\mathrm{a}}=1 \times 10^{8}$, $M=1.25 \times 10^{-8}, \bar{\eta}=1 \times 10^{5}$ and $\epsilon=1$. The fast diffuser hop rate is four times larger than that of the slow diffuser.

in section 4.1, the error for the diffusion equation should be less than $1 \%$. Figure 10 shows snapshots of the mole fraction profiles (left column) and velocity fields (right column) from a 2D simulation. As the fast diffuser diffuses from the right to the left side, the vacancy elimination and generation cause contraction and expansion on the right and left sides, respectively. As a result, the initially rectangular slab deforms into a bottle-shaped object as diffusion proceeds. 


\subsection{Thermal stress}

SOFCs usually operate at temperatures near $500-1000^{\circ} \mathrm{C}$. Evaluating the thermal stress resulting from the differences in thermal expansion and elastic moduli is important for analyzing mechanical failure. We expand the generalized mechanical equilibrium equation (17) for a linear, elastic and isotropic solid. (Note that the derivation for the mechanical equilibrium equation is general and is not limited to isotropic solids. We selected an isotropic model because of the lack of available crystallographic information among the experimental data.) The equation is discretized in a central finite difference scheme and numerically solved by an alternating-direction line-relaxation (ADLR) solver; see appendix G for details.

The thermal expansion rates of the ceramic electrolyte (GDC) and cathode (LSC) are taken to be $12.3 \times 10^{-6} \mathrm{~K}^{-1}[73]$ and $10.6 \times 10^{-6} \mathrm{~K}^{-1}$ [74], such that the thermal expansions at operation temperature are 0.0123 and 0.0106 , respectively. (Here, we have assumed arbitrarily that the composite material is relaxed at a reference temperature, and assumed an operation temperature of $1000{ }^{\circ} \mathrm{C}$ above the reference temperature.) We chose the elastic constants of GDC to be isotropic $\left(\lambda_{11}-\lambda_{12}=2 \lambda_{44}\right)$, and the values are $\lambda_{11}^{\mathrm{GDC}}=375.94 \mathrm{GPa}$, $\lambda_{12}^{\mathrm{GDC}}=188.54 \mathrm{GPa}$ and $\lambda_{44}^{\mathrm{GDC}}=93.70 \mathrm{GPa}$, calculated from Young's modulus of $250 \mathrm{GPa}$ and Poisson's ratio of 0.334 [75, 76]; see appendix G. The LSC phase is softer than the GDC phase, and its elastic constant is also assumed to be isotropic. The values are selected to be $\lambda_{11}^{\mathrm{LSC}}=269.23 \mathrm{GPa}, \lambda_{12}^{\mathrm{LSC}}=115.38 \mathrm{GPa}$ and $\lambda_{44}^{\mathrm{LSC}}=76.29 \mathrm{GPa}$, based on Young's modulus of $200 \mathrm{GPa}$ and Poisson's ratio of 0.3 [74]. As in section 5.1, we again use domain parameters to indicate the GDC phase ( $\psi_{1}=1$ inside the GDC and $\psi_{1}=0$ outside the GDC) and the LSC phase ( $\psi_{2}=1$ inside the LSC and $\psi_{2}=0$ outside the LSC). The entire solid phase is then represented by the sum of the two phases, $\psi=\psi_{1}+\psi_{2}=1$. The body force term and elastic constant tensor are replaced by an interpolated, spatially dependent thermal expansion and elastic constant tensor according to the domain parameters; see appendix G. The solid surface is assumed to be traction-free, $N_{i}=0$.

In this simulation, we selected the same computational box as in the case of figure $9(a)$, containing $321 \times 261 \times 297$ grid points in the $x, y$ and $z$ directions, respectively. Each grid point represents a voxel in the experimentally obtained microstructure. The yellow color indicates the LSC phase, and the semitransparent cyan color indicates the GDC phase. The same values of $\mathcal{R}_{1}$ and $\mathcal{R}_{2}$ as in section 5.1 were used. The grid spacing is $\Delta x=25 \mathrm{~nm}$, such that the computational box spans $8.025 \times 6.524 \times 7.425 \mu \mathrm{m}^{3}$. We assumed a rigid computational box with frictionless boundaries on the six sides, which means that $u=\partial v / \partial x=\partial w / \partial x=0$ on the two $y-z$ planes, $v=\partial u / \partial y=\partial w / \partial y=0$ on the two $x-z$ planes, and $w=\partial u / \partial z=\partial v / \partial z=0$ on the two $x-y$ planes of the computational box boundaries, where $u, v$ and $w$ are the displacements along the $x$-, $y$ - and $z$-axes, respectively. While this set of boundary conditions is not realistic for SOFC material environment, we chose it for the demonstration purpose in order to avoid overlaps with a future publication of physically based SOFC simulations.

Shown in figure 11 $(a)$ are the calculated mean stress distributions resulting from thermal expansion in a confined sample. The mean stress is defined by $\sigma_{m}=\left(\sigma_{x x}+\sigma_{y y}+\sigma_{z z}\right) / 3$, where the stress components are calculated according to the method provided in appendix G. Here, we choose mean stress to illustrate the effective pressure in the solid. A negative mean stress indicates that the region is under compression. Despite a complicated stress distribution observed because of the complex geometry, the overall magnitude of the mean stress is roughly between 2 and $4 \mathrm{GPa}$, which can be roughly estimated by the product of Young's modulus and the thermal expansion with an enhancement resulting from the porosity of the solid. Additionally, an overall larger stress in the GDC phase is observed, reflecting that the GDC 


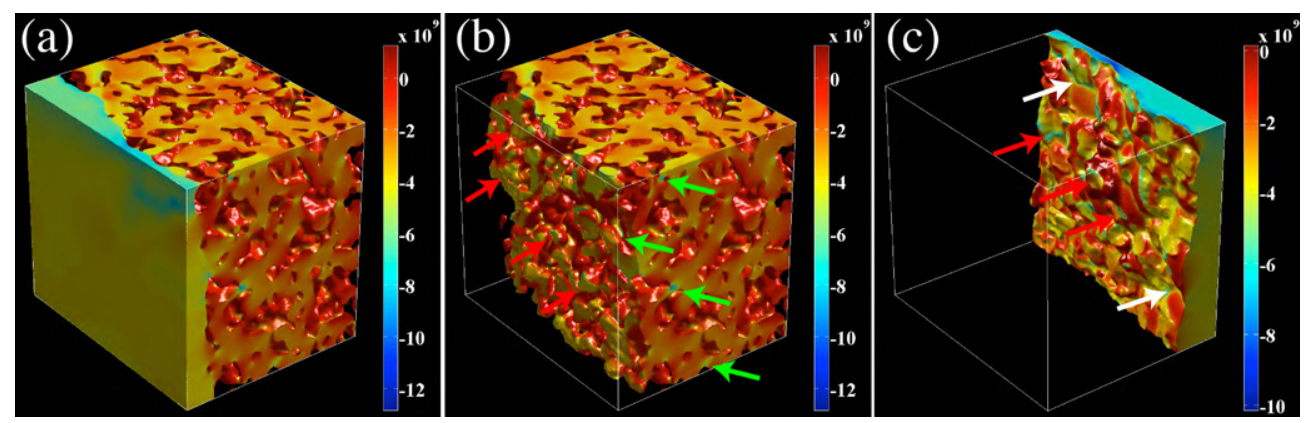

Figure 11. The mean stresses resulting from thermal expansion in $(a)$ the entire solid phase, $(b)$ the cathode phase, and $(c)$ the electrolyte phase after rotating the volume $180^{\circ}$ around the $z$-axis. The unit of stress is $\mathrm{Pa}$.

phase is harder than the LSC phase. Figure 11(b) shows the mean stress in the LSC phase and figure 11(c) shows the mean stress on the GDC surface after rotating the volume $180^{\circ}$ around the $z$-axis. Three types of stress enhancements can be observed in the simulation result. At the cathode-electrolyte contacting surfaces, stress is enhanced because of the mismatch of thermal expansion and elastic constants between the two materials; see the red arrows in figures 11(b) and $(c)$. The second is the concentrated stress observed at the grooves on the electrolyte surface (not contacting the cathode), as shown by the white arrows in figure 11(c). The third type is the stress concentration effect at the bottlenecks in the cathode phase, where the stresses are larger by roughly a factor of three to four compared with the overall value, as shown by the green arrows in figure 11(b). The simulation results demonstrate that the SBM can properly capture the linear elasticity and the geometric effects of the system based on a diffuse-interface defined geometry.

\subsection{Phase transformations in the presence of a foreign surface}

The Allen-Cahn equation describes the dynamics of a nonconserved order parameter, which can be taken as a model for the ordering of magnetic moments [40] and diffusionless phase transformations that involve only changes in crystalline order [40,77] or ordering in other systems, for example in [78]. This equation can also be used as a model for evaporationcondensation dynamics [40,41]. Here, we use the Allen-Cahn equation to examine the evaporation of a droplet on a rough surface. The domain parameter was given a ripplelike feature, as shown in figure 12, having a hyperbolic-tangent-like profile continuously transitioning through the substrate surface $(\psi=1$ above the surface, and $\psi=0$ below the surface). The droplet phase was placed on top of the boundary, and its shape was evolved by the SBM formulation of the Allen-Cahn equation (22) using the standard central difference scheme in space and an Euler explicit scheme in time. The simulation was performed in two dimensions, using the parameters $\Delta x=1, M=1$ and $\epsilon=1$, with a domain size of $L_{x}=100$ and $L_{y}=100$. The contact angle was set at $135^{\circ}$, and a zero-gradient boundary condition of $\phi$ is set at the computational box boundaries.

The evolution of the droplet surface as it evaporates is illustrated in figure $12(a)$ as a contour $(\phi=0.5)$ plotted at equal intervals of 270 dimensionless time units. The color change from blue to red indicates various times from the initial to the final stages, respectively. As the surface evolves, it is clear that the contact angle is maintained, as shown in figure $12(b)$. The dynamics of the motion of the three-phase boundary are interesting in that the velocity 


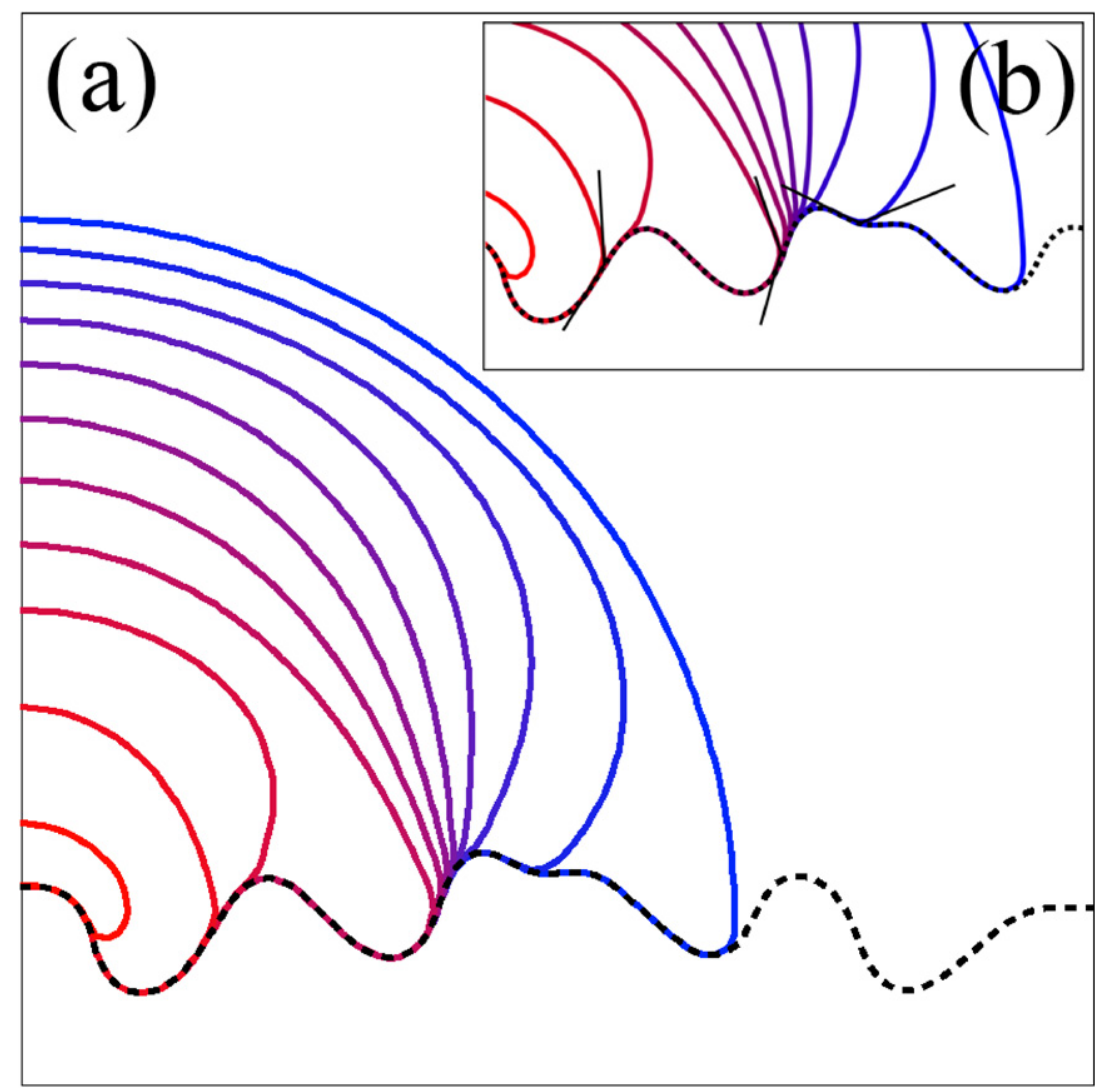

Figure 12. (a) The evolution of an evaporating droplet on a rough surface (dashed line) governed by Allen-Cahn dynamics. The contact angle between the droplet and the surface is imposed at $135^{\circ}$. Solid curves of various colors represent the profile of the droplet at different times. The outermost blue line represents the initial state, and the innermost red line represents the final state (recorded before complete evaporation in the simulation); the lines are plotted at time intervals of 270 dimensionless time units. The velocity of the three-phase boundary is considerably affected by the surface profile. (b) A magnified view of the three-phase boundary, showing that the contact angle is accurately set. The angle made by the thin black lines is $135^{\circ}$.

changes depending on the angle of the surface (with respect to the horizontal axis), which can be inferred from the change in the density of the contours. Because the interfacial energy is assumed to be constant, the droplet would prefer to have a circular cap shape. However, the contact angle imposes another constraint at the three-phase boundary. When the orientation of the surface is such that both of these conditions are nearly met, the motion of the three-phase boundary is slow as the droplet evaporates. When the orientation becomes such that the shape of the droplet near the three-phase boundary must be deformed (compared with the circular cap), the three-phase boundary moves very quickly, which leads to an unsteady motion of the three-phase boundary. In contrast, at the top of the droplet far from the substrate, the curvature is barely affected by the angle of the substrate surface; thus, the phase interface there moves at a speed inversely proportional to the radius.

Next, we conducted a 3D simulation of the dynamics of relaxation of an initially hemispherical droplet on a rough surface. Assuming the droplet volume is conserved, we evolve equation (23) for this simulation. The contact-angle boundary condition imposed at the 

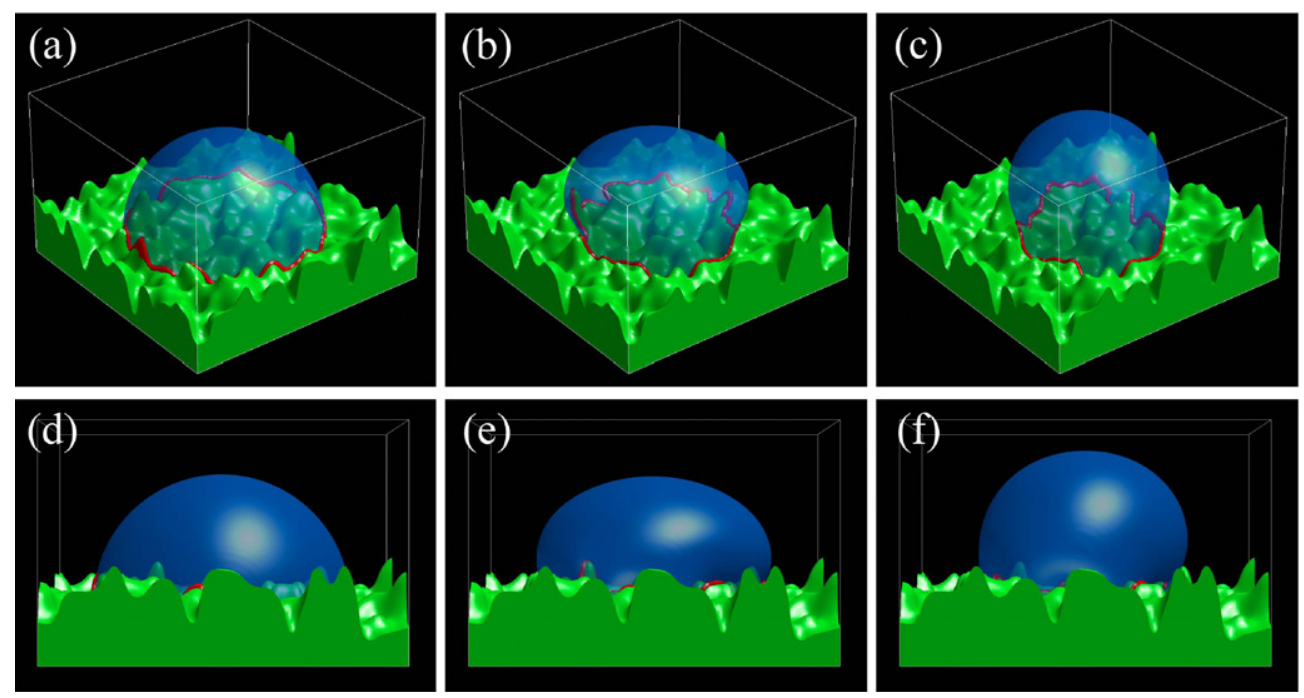

Figure 13. A droplet relaxing toward its equilibrium shape. The evolution is modeled by the Cahn-Hilliard equation with a contact angle of $135^{\circ}$ to the irregular substrate surface: $(a)$ initial $(t=0),(b)$ intermediate $\left(t=3 \times 10^{3}\right)$ and $(c)$ equilibrium $\left(t=2.35 \times 10^{4}\right)$ states. The threephase boundaries are delineated in red. Side views of the droplet are shown in $(d),(e)$ and $(f)$, corresponding to $(a),(b)$ and $(c)$, respectively.

three-phase boundary is $135^{\circ}$. The computational box sizes are $L_{x}=L_{y}=120$ and $L_{z}=80$. By setting $\zeta=1$ in the hyperbolic tangent function that describes the surface, the domaininterface thickness is $\xi_{0}=4.185$, which leads to $\mathcal{R}_{2}=4.185$ for this simulation. Here, surface features with curvatures up to a few times $\xi_{0}$ are properly represented. However, since the rough surface was generated randomly, features exist that are not fully captured by this parameter set; such features are effectively smoothed out over the interfacial thickness in the simulation. Figure 13 shows the snapshots of the droplet-shape evolution process. The droplet changes its shape to satisfy the imposed contact angle, and the droplet evolves into a shape for which the total surface energy is minimized. The behavior favoring dewetting imposed by the contact angle $\left(\theta>90^{\circ}\right)$ is properly reflected in the lifting of the droplet, as shown in figures $13(a)-(c)$ and $(d)-(f)$. During this relaxation process, the three-phase boundary moves toward the center as the droplet-substrate contacting area decreases, as shown in figures 13(a)$(c)$. This model and numerical method has been applied to simulate a nickel particle coarsening process in the complex channel within supporting porous ceramic microstructure (consisting of yttria-stabilized zirconia) in SOFC anodes, and to estimate the degradation of the anode material during SOFC operation [79].

\section{Discussion and conclusions}

In this work, we demonstrated a generalized formulation of the SBM. This method allows Neumann, Dirichlet or mixed boundary conditions to be imposed on a diffuse interface for solving partial differential equations within the region where the domain parameter $\psi$ uniformly equals 1 . The derivation of the method, as well as its implementation, is straightforward. The method can be used to solve differential equations numerically without complicated and time-consuming structural meshing of the domain of interest, as the domain 
boundary is specified by a spatially varying function. Instead, any grid system, including a regular Cartesian grid system, can be used with this method.

This SBM approach is flexible in coupling multiple differential equations. In section 3.3, we demonstrated how this method can be used to couple bulk diffusion with surface reactiondiffusion into a single equation while the two equations serve as complementary boundary conditions. In principle, this method can be used to couple multiple differential equations in different regions defined by different domain parameters. For example, if the physics within a domain defined by $\psi_{i}=1$ are governed by a differential equation $H_{i}$, the overall phenomenon will be then represented by $H=\sum_{i} \psi_{i} H_{i}$, where the subscript ' $i$ ' denotes the $i$ th domain and $\sum_{i} \psi_{i}=1$ represents the entire computational box. When sharing the diffuse interfaces between domains, the physical quantities can be interconnected as boundary conditions for each equation in each domain. Therefore, this method could be used to simulate coupled multiphysical and/or multiple-domain problems such as fluid-solid interaction phenomena or diffusion in multi-material polycrystalline solids.

We further demonstrated the capability of applying the SBM to moving boundary problems in section 5.2. When the locations of domain boundaries are updated by phase-field-type dynamics such that the domain parameters remain uniformly at 1 and 0 on either side of the interface, the SBM can be conveniently employed to solve partial differential equations with moving boundaries. In addition to the phase-field-type dynamics, the SBM is also applicable to moving boundary problems implementing the level set method $[34,35,80]$, with the domain parameter obtained simply by taking the hyperbolic tangent of the distance function.

In addition to Neumann and Dirichlet boundary conditions, we also showed the capability of the SBM for specifying contact angles between phase boundaries and domain boundaries (sections 3.5, 4.3 and 5.4). This type of boundary condition is difficult to impose using conventional sharp-interface models.

Although the SBM has many advantages, we have also shown in section 4 and appendix A that the nature of the diffuse interface inevitably introduces an error proportional to the interfacial thickness because we expand an originally zero-thickness boundary into a finitethickness interface. In particular, the error increases when the characteristic lengths in the system are not sufficiently larger than the interfacial thickness. To ensure an acceptable accuracy in a calculation involving a complex microstructure, one will thus need to statistically examine the spatially varying characteristic lengths (such as the radius of the curvature), and set the interfacial thickness such that a large fraction of the microstructural features have sufficiently larger radii of curvatures than the interfacial thickness. The diffuse interface also leads to another error source depending on the resolution of the rapid transition of the domain parameter across the interfacial region. When numerically solving the SBM formulated equations, properly capturing the gradient of the domain parameter across the interface becomes very important. Based on our experience, three to five grid points are necessary to properly resolve the diffuse interfaces so that the errors are well-controlled. Moreover, when solving time-dependent equations, a singularity arises because of the terms $1 / \psi$ and $1 / \psi^{2}$ used to impose the Neumann and Dirichlet boundary conditions, respectively. In practice, a small value is necessarily added to $\psi$ to avoid singularity resulting from division by zero. In our simulations, the errors were quickly saturated when the value added to $\psi$ was selected to be smaller than $1 \times 10^{-5}$ when three to five grid spacings were used for the interfacial regions, which suggests it is unnecessary to select a smaller value for the singularity-control term. However, when solving time-independent equations, such as the mechanical equilibrium equation and the steady-state diffusion equation, there are no singular terms in the equations. The small additional term is then merely used to condition the matrix solver. In this case, it can be on the order of numerical precision, such as $1 \times 10^{-16}$. 
Based on the general nature of the derivation, the SBM is applicable to generalized boundary conditions, including time-dependent boundary values important for simulating the evolution of many physical systems. Because the domain boundaries are not specifically defined in the SBM, this method can be applied to almost any geometry as long as it can be defined by the domain parameter. The developed method is thus a very powerful and convenient technique for solving differential equations in complex geometries that are often difficult and time-consuming to structurally mesh. As 3D image-based calculations are increasingly prevalent in scientific and engineering research fields [81-83], where voxelated data from serial scanning or sectioning are often utilized and are difficult to render as meshes, the SBM is expected to be widely employed to simulate and study physics in complex geometries defined by $2 \mathrm{D}$ pixelated and 3D voxelated data with a simple process of smoothing the domain boundaries.

\section{Acknowledgments}

HCY and KT thank the National Science Foundation for financial support under grant Nos. 0511232, 0502737 and 0854905. HYC and KT thank the National Science Foundation for financial support under grant Nos. 0542619 and 0907030. KT also acknowledges the support of the National Science Foundation under grant No. 0746424. The authors thank John Lowengrub, Axel Voigt, Xiaofan Li, Anton Van der Ven and James Warren for valuable discussions and comments. The authors also thank Scott Barnett and Stuart Adler for providing the experimental 3D microstructures used in the demonstration.

\section{Appendix A. Proof of convergence for Neumann and Dirichlet boundary conditions for the diffusion equation}

To demonstrate that the SBM formulated diffusion equation satisfies the assigned Neumann boundary condition (specifying the boundary flux or normal gradient), we use the 1D version of equation (6) without loss of generality for cases with higher dimensions. By reorganizing terms and integrating over the interfacial region, we obtain

$$
\int_{a_{i}-\xi / 2}^{a_{i}+\xi / 2} \psi\left(\frac{\partial C}{\partial t}-S\right) \mathrm{d} x=\left.\psi D \frac{\partial C}{\partial x}\right|_{a_{i}-\xi / 2} ^{a_{i}+\xi / 2}-\int_{a_{i}-\xi / 2}^{a_{i}+\xi / 2}\left|\frac{\partial \psi}{\partial x}\right| D B_{\mathrm{N}} \mathrm{d} x,
$$

where $a_{i}-\xi / 2<x<a_{i}+\xi / 2$ is the region of the interface and $\xi$ is the thickness of the interface. Following [2,3,22,25], we introduce the mean value theorem of integrals, which states that for a continuous function $g(x)$ a constant value, $h_{0}$, exists such that $\min g(x)<\int_{p}^{q} g(x) \mathrm{d} x /(q-p)=h_{0}<\max g(x)$, where $p<x<q$. By eliminating the second terms on the right-hand sides of equations (6) and (A.1), the no-flux boundary condition can be imposed $\left(B_{\mathrm{N}}=0\right)$; the resulting equation is similar to those proposed in $[1-3,22,25]$. However, here we retain the term to maintain the generality of the method. Therefore, the analysis presented herein leads to an extension of the original method that significantly extends its applicability.

Because the function on the left-hand side of equation (A.1) is continuous and finite within the interfacial region, we can relate its value to the interfacial thickness by $h_{0} \xi$, according to the mean value theorem of integrals. Using the conditions that $\psi=1$ at $x=a_{i}+\xi / 2$ and $\psi=0$ at $x=a_{i}-\xi / 2$, the first term on the right-hand side of equation (A.1) is written as $D(\partial C / \partial x)_{a_{i}+\xi / 2}$. Because $|\partial \psi / \partial x|=0$ for $x<a_{i}-\xi / 2$ or $x>a_{i}+\xi / 2$, the bounds of the 
integral can be extended to $-\infty$ and $\infty$. Therefore, we can rewrite equation (A.1) as

$$
h_{0} \xi=\left.D \frac{\partial C}{\partial x}\right|_{a_{i}+\xi / 2}-\int_{-\infty}^{+\infty}\left|\frac{\partial \psi}{\partial x}\right| D B_{\mathrm{N}} \mathrm{d} x
$$

and by taking the limit of this expression as $\xi \rightarrow 0$, we obtain

$$
\left.D \frac{\partial C}{\partial x}\right|_{a_{i}}=\int_{-\infty}^{+\infty} \delta\left(x-a_{i}\right) D B_{\mathrm{N}} \mathrm{d} x=\left.D B_{\mathrm{N}}\right|_{a_{i}},
$$

where $\partial C /\left.\partial x\right|_{a_{i}+\xi / 2}=\partial C /\left.\partial x\right|_{a_{i}}$ and $\lim _{\xi \rightarrow 0}|\partial \psi / \partial x|=\delta\left(x-a_{i}\right)$ when $\psi$ takes the form of a hyperbolic tangent function and $\delta\left(x-a_{i}\right)$ is the Dirac delta function. The Dirac delta function has the property that $\int_{-\infty}^{+\infty} \delta\left(x-a_{i}\right) f(x) \mathrm{d} x=f\left(a_{i}\right)$, providing the second equality in equation (A.3). Therefore, equation (A.3) clearly shows that the SBM recovers the Neumann boundary condition at the boundary when the thickness of the diffuse boundary approaches zero. This convergence has been observed for both stationary and moving boundaries [22, 34, 35].

To demonstrate the convergence of the solution at the boundaries to the specified boundary value, we again use a $1 \mathrm{D}$ version of the SBM formulated equation. Integrating equation (7) over the interfacial region and reorganizing terms, we obtain

$$
\begin{aligned}
\int_{a_{i}-\xi / 2}^{a_{i}+\xi / 2}\left[\psi^{2} \frac{\partial C}{\partial t}\right. & \left.-\psi \frac{\partial}{\partial x}\left(\psi D \frac{\partial C}{\partial x}\right)-\psi^{2} S\right] \mathrm{d} x \\
& =-\int_{a_{i}-\xi / 2}^{a_{i}+\xi / 2} D\left(\frac{\partial \psi}{\partial x}\right)\left[\frac{\partial \psi C}{\partial x}-B_{\mathrm{D}} \frac{\partial \psi}{\partial x}\right] \mathrm{d} x
\end{aligned}
$$

Similar to the derivation of equation (A.3), the left-hand side of equation (A.4) is proportional to the interfacial thickness and approaches zero in the limit of $\xi \rightarrow 0$. On the right-hand side of equation (A.4), the gradient of $\psi$ approaches the Dirac delta function, $\delta\left(x-a_{i}\right)$, as the interface thickness approaches zero. Therefore, we can reduce equation (A.4) to $\lim _{\xi \rightarrow 0} h_{0} \xi=-D\left[\partial(\psi C) / \partial x-B_{\mathrm{D}} \partial \psi / \partial x\right]$ in the limit $\xi \rightarrow 0$. By integrating over the interfacial region again, we obtain

$$
-\lim _{\xi \rightarrow 0} \frac{h_{0} \xi^{2}}{D}=\left.C\right|_{a_{i}+\xi / 2}-\int_{a_{i}-\xi / 2}^{a_{i}+\xi / 2} B_{\mathrm{D}} \frac{\partial \psi}{\partial x} \mathrm{~d} x
$$

which gives $\left.C\right|_{a_{i}}=\left.B_{\mathrm{D}}\right|_{a_{i}}$ in the limit of $\xi \rightarrow 0$ because $\left.C\right|_{a_{i}+\xi / 2}=\left.C\right|_{a_{i}}$ and $\lim _{\xi \rightarrow 0}(\partial \psi / \partial x)=$ $\delta\left(x-a_{i}\right)$. Therefore, the SBM formulation recovers the specified Dirichlet boundary condition: $C=B_{\mathrm{D}}$ at $x=a_{i}$.

\section{Appendix B. Surface Laplacian operator and discretization scheme for solving coupled surface diffusion, reaction and Bulk diffusion equation}

The surface gradient operator is defined by

$$
\nabla_{\mathrm{s}}=(\boldsymbol{I}-\boldsymbol{n} \otimes \boldsymbol{n}) \nabla=\left(\begin{array}{ccc}
1-n_{1} n_{1} & -n_{1} n_{2} & -n_{1} n_{3} \\
-n_{2} n_{1} & 1-n_{2} n_{2} & -n_{2} n_{3} \\
-n_{3} n_{1} & -n_{3} n_{2} & 1-n_{3} n_{3}
\end{array}\right)\left[\begin{array}{l}
\partial / \partial x_{1} \\
\partial / \partial x_{2} \\
\partial / \partial x_{3}
\end{array}\right]
$$

where $n_{i}$ is the $i$ th component of the inward unit normal vector (here, $i=1,2$ and 3 , corresponding to the $x, y$ and $z$ directions, respectively). In tensor notation, this operator can be written as $\nabla_{\mathrm{s}}=m_{i j} \partial / \partial x_{j}$. The repeated indices indicate summation over the index. The coefficients $m_{i j}$ are related to the surface unit normal by $m_{11}=1-n_{1} n_{1}, m_{22}=1-n_{2} n_{2}$, 
$m_{33}=1-n_{3} n_{3}, m_{12}=m_{21}=-n_{1} n_{2}, m_{13}=m_{31}=-n_{1} n_{3}$ and $m_{23}=m_{32}=-n_{2} n_{3}$. The surface Laplacian operator is defined by the surface divergence of the surface gradient:

$$
\nabla_{\mathrm{s}}^{2}=\nabla_{\mathrm{s}} \cdot \nabla_{\mathrm{s}}=m_{i j} \frac{\partial}{\partial x_{j}}\left(m_{i k} \frac{\partial}{\partial x_{k}}\right) \text {. }
$$

The scalar surface Laplacian is a sum of nine second-order-partial-differential-operator (diagonal) terms (where $j=k$ ) and 18 mixed-partial (cross) terms of the differential operator (where $j \neq k$ ).

The 'diagonal' terms can be discretized by central difference schemes similar to

$\frac{\partial}{\partial x}\left(m \frac{\partial C}{\partial x}\right)=\frac{1}{\Delta x}\left(m_{i+1 / 2, j, k} \frac{C_{i+1, j, k}-C_{i, j, k}}{\Delta x}-m_{i-1 / 2, j, k} \frac{C_{i, j, k}-C_{i-1, j, k}}{\Delta x}\right)$,

and $m_{i+1 / 2, j, k}=\left(m_{i+1, j, k}+m_{i, j, k}\right) / 2$. The discretization scheme along the $y$ and $z$ axes can be similarly obtained. The cross operator can be calculated using the following discretization scheme: for example, the $\partial(m \partial C / \partial y) \partial x$ can be discretized as

$$
\frac{\partial}{\partial x}\left(m \frac{\partial C}{\partial y}\right)=\frac{1}{2 \Delta x}\left(m_{i+1, j, k} \frac{C_{i+1, j+1, k}-C_{i+1, j-1, k}}{2 \Delta y}-m_{i-1, j, k} \frac{C_{i-1, j+1, k}-C_{i-1, j-1, k}}{2 \Delta y}\right),
$$

and similarly for other components.

For the simulations of oxygen-vacancy diffusion in a cylinder in section 4.2 , we consider a cylindrical symmetry for the differential operator, in which the dimensions reduce to effectively $2 \mathrm{D}$, such that the 'bulk' term in equation (12) becomes

$$
\nabla \cdot(\psi \nabla C)=\frac{1}{r} \frac{\partial}{\partial r}\left(r \psi \frac{\partial C}{\partial r}\right)+\frac{\partial}{\partial z}\left(\psi \frac{\partial C}{\partial z}\right),
$$

with only components in the radial and axial directions. Here, we have set $D_{\mathrm{b}}$ at 1 for clarity of the derivation. The first term on the right-hand side can be rewritten and discretized using the central difference scheme as

$$
\begin{gathered}
\frac{2}{\partial\left(r^{2}\right)} \partial\left(r \psi \frac{\partial C}{\partial r}\right)=\frac{2}{r_{i+1 / 2, j}^{2}-r_{i-1 / 2, j}^{2}}\left(r_{i+1 / 2, j} \psi_{i+1 / 2, j} \frac{C_{i+1, j}-C_{i, j}}{r_{i+1, j}-r_{i, j}}\right. \\
\left.-r_{i-1 / 2, j} \psi_{i-1 / 2, j} \frac{C_{i, j}-C_{i-1, j}}{r_{i, j}-r_{i-1, j}}\right),
\end{gathered}
$$

where the subscript $i$ and $j$ denote the $i$ th and $j$ th grid points in the radial and axial directions, respectively. If the radial grid spacing is selected to be uniform, $r_{i+1, j}-r_{i, j}=r_{i, j}-r_{i-1, j}=\Delta r$. The second term on the right-hand side of equation (B.5) can be discretized using the scheme provided in equation (B.3).

The surface Laplacian with cylindrical symmetry is given as $\nabla_{\mathrm{s}}^{2} C=$

$m_{r r} \frac{1}{r} \frac{\partial}{\partial r}\left(r m_{r r} \frac{\partial C}{\partial r}\right)+m_{r r} \frac{\partial}{\partial r}\left(m_{r z} \frac{\partial C}{\partial z}\right)+m_{r z} \frac{\partial}{\partial z}\left(m_{r r} \frac{\partial C}{\partial r}\right)+m_{r z} \frac{\partial}{\partial z}\left(m_{r z} \frac{\partial C}{\partial z}\right)$

$+m_{z r} \frac{1}{r} \frac{\partial}{\partial r}\left(r m_{z r} \frac{\partial C}{\partial r}\right)+m_{z r} \frac{\partial}{\partial r}\left(m_{z z} \frac{\partial C}{\partial z}\right)+m_{z z} \frac{\partial}{\partial z}\left(m_{z r} \frac{\partial C}{\partial r}\right)+m_{z z} \frac{\partial}{\partial z}\left(m_{z z} \frac{\partial C}{\partial z}\right)$,

where $m_{r r}=1-n_{r} n_{r}, m_{r z}=m_{z r}=-n_{r} n_{z}$, and $m_{z z}=1-n_{z} n_{z}$. The 'diagonal' terms in the radial and axial directions can be discretized in a manner similar to equations (B.6) and (B.3), respectively, and the 'cross' terms can be discretized as in equation (B.4) for solving equation (12) with an additional factor of $m$. 


\section{Appendix C. Derivation of the mechanical equilibrium equation}

To perform the SBM formulation on the tensorial mechanical equilibrium equation, we multiply equation (14) by $\psi$ and use the mathematical identity $\psi\left(\partial H_{i j} / \partial x_{j}\right)=\partial\left(\psi H_{i j}\right) / \partial x_{j}-$ $\left(\partial \psi / \partial x_{j}\right) H_{i j}$ to obtain

$$
\begin{gathered}
\frac{\partial}{\partial x_{j}}\left[\psi C_{i j k l} \frac{1}{2}\left(\frac{\partial u_{k}}{\partial x_{l}}+\frac{\partial u_{l}}{\partial x_{k}}\right)\right]-\frac{\partial \psi}{\partial x_{j}} C_{i j k l} \frac{1}{2}\left(\frac{\partial u_{k}}{\partial x_{l}}+\frac{\partial u_{l}}{\partial x_{k}}\right) \\
=\frac{\partial}{\partial x_{j}}\left(\psi \rho C_{i j k l} \delta_{k l}\right)-\frac{\partial \psi}{\partial x_{j}} \rho C_{i j k l} \delta_{k l} .
\end{gathered}
$$

By collecting the terms associated with $\partial \psi / \partial x_{j}$, we obtain equation (15) as given in section 3.4.

To impose a Dirichlet boundary condition (a specified displacement) on the mechanical equilibrium equation, we multiply the left-hand side of equation (14) by $\psi^{2}$ to obtain

$$
\begin{gathered}
\psi^{2} \frac{\partial}{\partial x_{j}} C_{i j k l} \frac{1}{2}\left(\frac{\partial u_{k}}{\partial x_{l}}+\frac{\partial u_{l}}{\partial x_{k}}\right)=\psi \frac{\partial}{\partial x_{j}}\left[\psi C_{i j k l} \frac{1}{2}\left(\frac{\partial u_{k}}{\partial x_{l}}+\frac{\partial u_{l}}{\partial x_{k}}\right)\right] \\
-\psi \frac{\partial \psi}{\partial x_{j}} C_{i j k l} \frac{1}{2}\left(\frac{\partial u_{k}}{\partial x_{l}}+\frac{\partial u_{l}}{\partial x_{k}}\right)
\end{gathered}
$$

where the second term on the right-hand side can be replaced by

$$
\begin{gathered}
\psi \frac{\partial \psi}{\partial x_{j}} C_{i j k l} \frac{1}{2}\left(\frac{\partial u_{k}}{\partial x_{l}}+\frac{\partial u_{l}}{\partial x_{k}}\right)=\frac{\partial \psi}{\partial x_{j}} C_{i j k l} \frac{1}{2}\left[\frac{\partial\left(\psi u_{k}\right)}{\partial x_{l}}+\frac{\partial\left(\psi u_{l}\right)}{\partial x_{k}}\right] \\
-\frac{\partial \psi}{\partial x_{j}} C_{i j k l} \frac{1}{2}\left(u_{k} \frac{\partial \psi}{\partial x_{l}}+u_{l} \frac{\partial \psi}{\partial x_{k}}\right)
\end{gathered}
$$

according to the product rule

$$
\begin{gathered}
\frac{\partial \psi}{\partial x_{j}} C_{i j k l}\left[\frac{\partial\left(\psi u_{k}\right)}{\partial x_{l}}+\frac{\partial\left(\psi u_{l}\right)}{\partial x_{k}}\right]=\frac{\partial \psi}{\partial x_{j}} C_{i j k l}\left[\left(\psi \frac{\partial u_{k}}{\partial x_{l}}+u_{k} \frac{\partial \psi}{\partial x_{l}}\right)+\left(\psi \frac{\partial u_{l}}{\partial x_{k}}+u_{l} \frac{\partial \psi}{\partial x_{k}}\right)\right] \\
=\psi \frac{\partial \psi}{\partial x_{j}} C_{i j k l}\left(\frac{\partial u_{k}}{\partial x_{l}}+\frac{\partial u_{l}}{\partial x_{k}}\right)+\frac{\partial \psi}{\partial x_{j}} C_{i j k l}\left(u_{k} \frac{\partial \psi}{\partial x_{l}}+u_{l} \frac{\partial \psi}{\partial x_{k}}\right) .
\end{gathered}
$$

Thereby, we obtain equation (18) in section 3.4.

\section{Appendix D. Relation used in the derivation of contact angle boundary condition}

Here, we multiply the equilibrium criterion of the phase-field model by $\nabla \phi$ to obtain

$$
\frac{\partial f}{\partial \phi} \nabla \phi-\left(\epsilon^{2} \nabla^{2} \phi\right) \nabla \phi=\nabla f-\frac{\epsilon^{2}}{2} \nabla(\nabla \phi)^{2}=0 .
$$

Integrating the above, we obtain $f-\epsilon^{2}|\nabla \phi|^{2} / 2=c_{1}$, where $c_{1}$ is a constant of integration. In the phase-field model, the order parameter remains at a uniform value in the bulk away from the interface; thus giving $|\nabla \phi|=0$ in the bulk. Therefore, $c_{1}$ is equal to the bulk value of $f$. For convenience, we have taken the free energy at the bulk values to be zero, and therefore $c_{1}=0$, leading to $\nabla \phi=\sqrt{2 f} / \epsilon$. However, the choice of the free energy value at the bulk is arbitrary, and therefore does not affect the result of the calculation as long as it is taken into account by replacing $f$ appearing in equation (20) by $f-c_{1}$. 


\section{Appendix E. Smoothing voxelated data using a distance function method}

The experimentally obtained microstructure is typically provided in a form of a 3D array containing voxels of different values indicating different phases. To incorporate the voxelated data into the SBM formulation, we must convert the discrete voxelated array into a domain parameter profile that continuously transitions from one phase to another. Here, we employ the distance function method commonly used for initialization in the level set method [44, 84]. First, we construct the sign function by assigning positive and negative values to the voxels in the solid and pore phases, respectively: $\operatorname{Sgn}(\boldsymbol{x})=1$ for the solid phase and $\operatorname{Sgn}(\boldsymbol{x})=-1$ for the pore phase, where $x$ is the position of a voxel. The distance function indicating the distance between the center of a voxel and the solid-pore interface is calculated by evolving the time-dependent equation $\partial \varphi(\boldsymbol{x}, t) / \partial t=\operatorname{Sgn}(\boldsymbol{x})(1-|\nabla \varphi(\boldsymbol{x}, t)|)$ to its equilibrium. This process is numerically implemented by

$\varphi(\boldsymbol{x}, t+\Delta t)= \begin{cases}\varphi(\boldsymbol{x}, t)+\Delta t[\operatorname{Sgn}(\boldsymbol{x})(1-|\nabla \varphi(\boldsymbol{x}, t)|)] & \text { if } \varphi(\boldsymbol{x}, t+\Delta t) \cdot \varphi(\boldsymbol{x}, t)>0 \\ \varphi(\boldsymbol{x}, t)+\Delta t[\operatorname{Sgn}(\boldsymbol{x}) v] & \text { if } \varphi(\boldsymbol{x}, t+\Delta t) \cdot \varphi(\boldsymbol{x}, t) \leqslant 0,\end{cases}$

where $v$ is a small nonzero value. The second case above prevents interfaces from moving more than one grid spacing by requiring that the sign of the function remains the same as the initial value. The absolute value of the gradient of the distance function is calculated using a Godunov upwind scheme [13, 85, 86]:

$$
\begin{aligned}
\left|\nabla \varphi_{i, j, k}\right|=[ & \max \left(\max \left(D_{x}^{+} \varphi_{i, j, k}, 0\right)^{2}, \max \left(-D_{x}^{-} \varphi_{i, j, k}, 0\right)^{2}\right) \\
& +\max \left(\max \left(D_{y}^{+} \varphi_{i, j, k}, 0\right)^{2}, \max \left(-D_{y}^{-} \varphi_{i, j, k}, 0\right)^{2}\right) \\
& \left.+\max \left(\max \left(D_{z}^{+} \varphi_{i, j, k}, 0\right)^{2}, \max \left(-D_{z}^{-} \varphi_{i, j, k}, 0\right)^{2}\right)\right]^{1 / 2},
\end{aligned}
$$

where $i, j$ and $k$ are the indices of the grid points along the $x, y$ and $z$ axes, respectively, and

$$
\begin{aligned}
& D_{x}^{+} \varphi_{i, j, k}=\left(\varphi_{i, j, k}-\varphi_{i-1, j, k}\right) / \Delta x, D_{x}^{-} \varphi_{i, j, k}=\left(\varphi_{i+1, j, k}-\varphi_{i, j, k}\right) / \Delta x, \\
& D_{y}^{+} \varphi_{i, j, k}=\left(\varphi_{i, j, k}-\varphi_{i, j-1, k}\right) / \Delta y, D_{y}^{-} \varphi_{i, j, k}=\left(\varphi_{i, j+1, k}-\varphi_{i, j, k}\right) / \Delta y, \\
& D_{z}^{+} \varphi_{i, j, k}=\left(\varphi_{i, j, k}-\varphi_{i, j, k-1}\right) / \Delta z, D_{z}^{-} \varphi_{i, j, k}=\left(\varphi_{i, j, k+1}-\varphi_{i, j, k}\right) / \Delta z .
\end{aligned}
$$

In practice, for the SBM, the function must take the form of the distance function only near the interfacial regions, and therefore the convergence condition can be placed in these regions only (and not in the bulk far from interfaces) as long as the values in the bulk are sufficiently large in magnitude.

From the distance function, we obtain a domain parameter based on the experimentally acquired voxelated data by taking the hyperbolic tangent of the distance function, $\psi(x)=$ $\{1+\tanh [\varphi(x) / \zeta]\} / 2$, where $\psi=0.5$ coincides the location of the zero level set $(\varphi=0)$, $\psi=1$ in the solid, $\psi=0$ in the pore, and the value of $\zeta$ controls the thickness of the interface.

\section{Appendix F. Projection method}

To simulate Kirkendall-effect-induced deformation, we model the solid diffusion couple as a very viscous fluid that deforms in a quasi-steady-state manner, namely, creep flow. In contrast, the environmental phase surrounding the solid is treated as a nearly inviscid fluid. A simple way to implement this model is to define the viscosity coefficient as $\eta(\psi)=\bar{\eta} \psi+v$, where $\bar{\eta}$ is a constant viscosity coefficient for the solid phase and $v \ll \bar{\eta}$ is a small value used to avoid numerical instability. To solve the velocity field with a variable viscosity coefficient, 
we adopt a projection method [67,68], in which the divergence of the viscous stress tensor is decomposed into a linear part and a residual part, giving

$$
\nabla \cdot \eta\left[\nabla \boldsymbol{v}+(\nabla \boldsymbol{v})^{\mathrm{T}}\right]=\Lambda \nabla \cdot\left[\nabla \boldsymbol{v}+(\nabla \boldsymbol{v})^{\mathrm{T}}\right]+\boldsymbol{r}_{\boldsymbol{v}}
$$

where $\Lambda$ is a constant scalar numerical parameter for the scheme (normally set between $0.5 \bar{\eta}$ and $\bar{\eta})$ and $\boldsymbol{r}_{\boldsymbol{v}}$ is a vector residual. Using the identity that $\nabla \cdot\left[\nabla \boldsymbol{v}+(\nabla \boldsymbol{v})^{\mathrm{T}}\right]=\nabla^{2} \boldsymbol{v}+\nabla(\nabla \cdot \boldsymbol{v})$, where $\nabla \cdot \boldsymbol{v}=g_{\mathrm{V}}$, and $\nabla^{2} \boldsymbol{v}=\partial^{2} v_{i} / \partial x_{j} \partial x_{j}$ is a vector containing the Laplacian of each velocity component, we rewrite equation $(29 a)$ as

$$
-\nabla P+\Lambda \nabla^{2} \boldsymbol{v}+\boldsymbol{r}_{\boldsymbol{v}}+\nabla\left(\Lambda-\frac{2 \eta}{d}\right) g_{\mathrm{V}}+\frac{1}{C_{\mathrm{a}}} \mu \nabla \psi=0 .
$$

By taking the divergence of equation (F.2), applying the relation $\nabla \cdot\left(\nabla^{2} v\right)=\nabla^{2}(\nabla \cdot v)=\nabla^{2} g_{\mathrm{V}}$ and rearranging the terms, we obtain the Poisson equation of the scalar pressure field, which serves as one of the two equations for the iterative scheme:

$$
\nabla^{2} P^{(n)}=\nabla \cdot \boldsymbol{r}_{\boldsymbol{v}}{ }^{(n-1)}+\nabla^{2}\left(2 \Lambda-\frac{2 \eta}{d}\right) g_{\mathrm{V}}+\frac{1}{C_{\mathrm{a}}} \nabla \cdot(\mu \nabla \psi),
$$

where the superscript $(n)$ denotes the $n$th iterative step. The second and third terms on the right-hand side are fixed during an evolution time step while the values of the velocity and pressure are updated during iteration.

For the velocity field, we reorganize equation (F.2) to obtain the Poisson equation for the velocity component in each coordinate direction:

$$
\nabla^{2} \boldsymbol{v}^{(n)}=\frac{1}{\Lambda}\left[\nabla P^{(n)}-\boldsymbol{r}_{\boldsymbol{v}}{ }^{(n-1)}-\nabla\left(\Lambda-\frac{2 \eta}{d}\right) g_{\mathrm{V}}-\frac{1}{C_{\mathrm{a}}} \mu \nabla \psi\right] .
$$

The residual vector $\boldsymbol{r}_{\boldsymbol{v}}$ is calculated using equation (F.1) and is updated during the iteration: $\boldsymbol{r}_{\boldsymbol{v}}^{(n)}=\nabla \cdot \eta\left[\nabla \boldsymbol{v}^{(n)}+\left(\nabla \boldsymbol{v}^{(n)}\right)^{\mathrm{T}}\right]-\Lambda \nabla^{2} \boldsymbol{v}^{(n)}-\Lambda \nabla g_{\mathrm{V}}$. The Poisson equations can be solved using an ADLR method similar to that described in appendix G, except that the operator is replaced here by a standard Laplacian operator. Within each time step for the deformation (equation (29c)) and diffusion (equation (31)) of the diffusion couple, the pressure and velocity fields are solved iteratively until the values of pressure and the velocity components converges. The convergence criteria is set to be $e \leqslant 1 \times 10^{-5}$, where $e$ is the relative error taken by dividing the rms difference between the values of two consecutive iterative steps by the average magnitude of the values in the previous step. The velocity field is then substituted into the advective terms in the order parameter and concentration evolution equations.

\section{Appendix G. Mechanical equilibrium equation and ADLR solver}

Here, we expand the generalized mechanical equilibrium equation (17) for a linear elastic and isotropic solid. In this case, the components of the elastic constant tensor are expressed by:

$$
\begin{aligned}
\lambda_{11} & =C_{1111}=C_{2222}=C_{3333}, \\
\lambda_{12} & =C_{1122}=C_{2211}=C_{2233}=C_{3322}=C_{3311}=C_{1133}, \\
\lambda_{44} & =C_{1212}=C_{1221}=C_{2112}=C_{2121}=C_{2323}=C_{2332} \\
& =C_{3223}=C_{3232}=C_{1313}=C_{1331}=C_{3113}=C_{3131} .
\end{aligned}
$$

The remaining elastic constant components vanish. For an isotropic solid, Young's modulus is related to Lame constants by $E=\lambda_{12}(1+v)(1-2 v) / v$, where $v$ is Poisson's ratio; the shear modulus is given by $\lambda_{44}=\lambda_{12}(1-2 \nu) / 2 \nu$, and the elastic constant $\lambda_{11}$ is given by $\lambda_{11}=\lambda_{12}+2 \lambda_{44}$. The pair, $\lambda_{12}$ and $v$, forms the set of Lame constants. 
We use coordinate notation to replace the indices $i=1,2$ and 3 with $x, y$ and $z$, respectively. With a traction-free boundary condition on the solid surface $\left(N_{i}=0\right)$, the SBM formulated mechanical equilibrium equation (17) can be written out for the $x, y$ and $z$ directions as

$$
\begin{gathered}
\frac{\partial}{\partial x}\left[\psi \lambda_{11}\left(\frac{\partial u}{\partial x}\right)\right]+\frac{\partial}{\partial y}\left[\psi \lambda_{44}\left(\frac{\partial u}{\partial y}\right)\right]+\frac{\partial}{\partial z}\left[\psi \lambda_{44}\left(\frac{\partial u}{\partial z}\right)\right]=\frac{\partial}{\partial x}\left[\psi \rho\left(\lambda_{11}+2 \lambda_{12}\right)\right] \\
-\frac{\partial}{\partial x}\left[\psi \lambda_{12}\left(\frac{\partial v}{\partial y}+\frac{\partial w}{\partial z}\right)\right]-\frac{\partial}{\partial y}\left[\psi \lambda_{44}\left(\frac{\partial v}{\partial x}\right)\right]-\frac{\partial}{\partial z}\left[\psi \lambda_{44}\left(\frac{\partial w}{\partial x}\right)\right], \\
\frac{\partial}{\partial x}\left[\psi \lambda_{44}\left(\frac{\partial v}{\partial x}\right)\right]+\frac{\partial}{\partial y}\left[\psi \lambda_{11}\left(\frac{\partial v}{\partial y}\right)\right]+\frac{\partial}{\partial z}\left[\psi \lambda_{44}\left(\frac{\partial v}{\partial z}\right)\right]=\frac{\partial}{\partial y}\left[\psi \rho\left(\lambda_{11}+2 \lambda_{12}\right)\right] \\
-\frac{\partial}{\partial x}\left[\psi \lambda_{44}\left(\frac{\partial u}{\partial y}\right)\right]-\frac{\partial}{\partial y}\left[\psi \lambda_{12}\left(\frac{\partial u}{\partial x}+\frac{\partial w}{\partial z}\right)\right]-\frac{\partial}{\partial z}\left[\psi \lambda_{44}\left(\frac{\partial w}{\partial y}\right)\right], \\
\frac{\partial}{\partial x}\left[\psi \lambda_{44}\left(\frac{\partial w}{\partial x}\right)\right]+\frac{\partial}{\partial y}\left[\psi \lambda_{44}\left(\frac{\partial w}{\partial y}\right)\right]+\frac{\partial}{\partial z}\left[\psi \lambda_{11}\left(\frac{\partial w}{\partial z}\right)\right]=\frac{\partial}{\partial z}\left[\psi \rho\left(\lambda_{11}+2 \lambda_{12}\right)\right] \\
-\frac{\partial}{\partial x}\left[\psi \lambda_{44}\left(\frac{\partial u}{\partial z}\right)\right]-\frac{\partial}{\partial y}\left[\psi \lambda_{44}\left(\frac{\partial v}{\partial z}\right)\right]-\frac{\partial}{\partial z}\left[\psi \lambda_{12}\left(\frac{\partial u}{\partial x}+\frac{\partial v}{\partial y}\right)\right],
\end{gathered}
$$

where $u, v$ and $w$ are the displacements along the $x$-, $y$ - and $z$-axes, respectively. Here, we provide the long form of the mechanical equilibrium equation for the sake of clarity for the readers.

To solve equations (G.2a)-(G.2c), we use an ADLR method as follows. We keep the 'diagonal' terms such as $\partial(\psi \lambda \partial / \partial x) / \partial x, \partial(\psi \lambda \partial / \partial y) / \partial y$ and $\partial(\psi \lambda \partial / \partial z) / \partial z$ on the left-hand sides, and move the 'cross' terms of the differential operator to the right-hand sides. We therefore obtain three equations with second-order-partial-differential operators for the three displacement components. The 'diagonal' terms on the left-hand sides can be discretized using the scheme in equation (B.3). The 'cross' terms moved to the right-hand sides can be calculated in a scheme similar to equation (B.4). Each equation in equations (G.2a)-(G.2c) can be represented by $\mathcal{L} \tilde{U}=\mathcal{S}$, where $\mathcal{L}$ is the linear operator on the left-hand side and $\mathcal{S}$ is the calculated right-hand side. This equation can be solved using an ADLR solver [87, 88] by decomposing the linear operator into the three axial directions:

$$
\begin{aligned}
& \mathcal{L}_{i+1, j, k} \tilde{U}_{i+1, j, k}^{(n+1 / 3)}-\mathcal{W}_{i, j, k} \tilde{U}_{i, j, k}^{(n+1 / 3)}+\mathcal{L}_{i-1, j, k} \tilde{U}_{i-1, j, k}^{(n+1 / 3)}=\mathcal{S}^{(n)}-\mathcal{L}_{y y} \tilde{U}_{y y}^{(n)}-\mathcal{L}_{z z} \tilde{U}_{z z}^{(n)} \\
& \mathcal{L}_{i, j+1, k} \tilde{U}_{i, j+1, k}^{(n+2 / 3)}-\mathcal{W}_{i, j, k} \tilde{U}_{i, j, k}^{(n+2 / 3)}+\mathcal{L}_{i, j-1, k} \tilde{U}_{i, j-1, k}^{(n+2 / 3)}=\mathcal{S}^{(n)}-\mathcal{L}_{x x} \tilde{U}_{x x}^{(n+1 / 3)}-\mathcal{L}_{z z} \tilde{U}_{z z}^{(n+1 / 3)} \\
& \mathcal{L}_{i, j, k+1} \tilde{U}_{i, j, k+1}^{(n+1)}-\mathcal{W}_{i, j, k} \tilde{U}_{i, j, k}^{(n+1)}+\mathcal{L}_{i, j, k-1} \tilde{U}_{i, j, k-1}^{(n+1)}=\mathcal{S}^{(n)}-\mathcal{L}_{x x} \tilde{U}_{x x}^{(n+2 / 3)}-\mathcal{L}_{y y} \tilde{U}_{y y}^{(n+2 / 3)}
\end{aligned}
$$

where $\mathcal{L}_{x x} \tilde{U}_{x x}=\mathcal{L}_{i+1, j, k} \tilde{U}_{i+1, j, k}+\mathcal{L}_{i-1, j, k} \tilde{U}_{i-1, j, k}, \mathcal{L}_{y y} \tilde{U}_{y y}=\mathcal{L}_{i, j+1, k} \tilde{U}_{i, j+1, k}+\mathcal{L}_{i, j-1, k} \tilde{U}_{i, j-1, k}$, $\mathcal{L}_{z z} \tilde{U}_{z z}=\mathcal{L}_{i, j, k+1} \tilde{U}_{i, j, k+1}+\mathcal{L}_{i, j, k-1} \tilde{U}_{i, j, k-1}$ and the superscript ( $n$ ) denotes the $n$th iterative step. Within each iterative step, we first solve along the $x$-axis using equation (G.3a), for which a simple tridiagonal matrix solver is employed for each column. Similarly, equations (G.3b) and (G.3c) are solved along the $y$ - and $z$-axes, respectively, with the updated value on the righthand sides. The above procedure is repeated until the solutions of equations (G.2a)-(G.2c) converge to their equilibrium values.

For the case in section 5.3, the solid phase $\left(\psi=\psi_{1}+\psi_{2}\right)$ includes two different materials: GDC $\left(\psi_{1}\right)$ and LSC $\left(\psi_{2}\right)$. Therefore, we smoothly interpolate material properties appearing 
in the differential operators: $\psi \lambda_{i j}=\psi_{1} \lambda_{i j}^{\mathrm{GDC}}+\psi_{2} \lambda_{i j}^{\mathrm{LSC}}$. Similarly, the body force term is interpolated by $\psi \rho\left(\lambda_{11}+2 \lambda_{12}\right)=\psi_{1} \rho^{\mathrm{GDC}}\left(\lambda_{11}^{\mathrm{GDC}}+2 \lambda_{12}^{\mathrm{GDC}}\right)+\psi_{2} \rho^{\mathrm{LSC}}\left(\lambda_{11}^{\mathrm{LSC}}+2 \lambda_{12}^{\mathrm{LSC}}\right)$, where $\rho=\alpha \Delta T$ is the thermal expansion. The thermal stress is calculated according to Hooke's law, which is written with the domain-parameter-interpolated elastic constants and thermal expansions as

$\sigma_{i j}=\left(\psi_{1} C_{i j k l}^{\mathrm{GDC}}+\psi_{2} C_{i j k l}^{\mathrm{LSC}}\right) \frac{1}{2}\left(\frac{\partial u_{k}}{\partial x_{l}}+\frac{\partial u_{l}}{\partial x_{k}}\right)-\left(\psi_{1} \rho^{\mathrm{GDC}} C_{i j k l}^{\mathrm{GDC}}+\psi_{2} \rho^{\mathrm{LSC}} C_{i j k l}^{\mathrm{LSC}}\right) \delta_{k l}$.

\section{References}

[1] Bueno-Orovio A 2006 Appl. Math. Comput. 183 813-8

[2] Bueno-Orovio A and Perez-Garcia V M 2006 Numer. Methods Partial Diff. Eqns 22 435-48

[3] Bueno-Orovio A, Perez-Garcia V M and Fenton F H 2006 SIAM J. Sci. Comput. 28 886-900

[4] Badea L and Daripa P 2001 SIAM J. Control. Optim. $40421-49$

[5] Peskin C S 2002 Acta Numer. 11 479-517

[6] Boyd J P 2005 Appl. Math. Comput. 161 591-7

[7] LeVeque R J and Li Z 1994 SIAM J. Numer. Anal. 31 1019-44

[8] Li Z and Ito K 2006 The Immersed Interface Method: Numerical Solutions of PDEs Involving Interfaces and Irregular Domains (Philadelphia, PA: SIAM)

[9] Lui S H 2009 J. Comput. Appl. Math. 225 541-57

[10] Sabetghadam F, Sharafatmandjoor S and Norouzi F 2009 J. Comput. Phys. 228 55-74

[11] Peskin C S and McQueen D M 1995 Symp. Soc. Exp. Biol. 49 265-76

[12] Tornberg A K and Engquist B 2003 J. Sci. Comput. 19 527-52

[13] Sethian J A 1999 Level Set Methods and Fast Marching Methods 2nd edn (Cambridge: Cambridge University Press)

[14] Adalsteinsson D and Sethian J A 2003 J. Comput. Phys. 185 271-88

[15] Sethian J A and Shan Y 2008 J. Comput. Phys. 227 6411-47

[16] Karma A and Rappel W-J 1998 Phys. Rev. E 57 4323-49

[17] Karma A and Rappel W-J 1999 Phys. Rev. E 60 3614-25

[18] Cahn J W 1977 J. Chem. Phys. 66 3667-72

[19] Granasy L, Pusztai T, Saylor D and Warren J A 2007 Phys. Rev. Lett. 98035703

[20] Warren J A, Pusztai T, Kornyei L and Granasy L 2009 Phys. Rev. B 79014204

[21] Qian T, Wang X-P and Ping S 2006 J. Fluid Mech. 564 333-60

[22] Kockelkoren J, Levine H and Rappel W-J 2003 Phys. Rev. E 68037702

[23] Levine H and Rappel W J 2005 Phys. Rev. E 72061912

[24] Fenton F H, Cherry E M, Karma A and Rappel W-J 2005 Chaos 15013502

[25] Buzzard G T, Fox J J and Siso-Nadal F 2007 SIAM J. Sci. Comput. 30 837-54

[26] Gal C G 2006 Math. Methods Appl. Sci. 29 2009-36

[27] Wu H 2007 Asymptotic Anal. 54 71-92

[28] Gal C G and Wu H 2008 Discrete Contin. Dyn. Syst. 22 1041-63

[29] Li X, Lowengrub J, Ratz A and Voigt A 2009 Commun. Math. Sci. 7 81-107

[30] Lowengrub J, Ratz A and Voigt A 2009 Phys. Rev. E 79031926

[31] Ratz A and Voigt A 2006 Commun. Math. Sci. 4 575-90

[32] Teigen K E, Li X R, Lowengrub J, Wang F and Voigt A 2009 Commun. Math. Sci. 7 1009-37

[33] Sohn J S, Tseng Y H, Li S W, Voigt A and Lowengrub J S 2010 J. Comput. Phys. 229 119-44

[34] Aland S, Lowengrub J and Voigt A 2010 CMES-Comput. Modeling Eng. Sci. 57 77-107

[35] Teigen K E, Song P, Lowengrub J and Voigt A 2011 J. Comput. Phys. 230 375-93

[36] Cahn J W and Hilliard J E 1958 J. Chem. Phys. 28 258-67

[37] Cahn J W and Hilliard J E 1959 J. Chem. Phys. 31 688-99

[38] Allen S M and Cahn J W 1979 Acta Metall. 27 1085-95

[39] Ginzburg V L and Landau L D 1950 Sov. Phys. JETP_USSR 201064.

[40] Chen L Q 2002 Annu. Rev. Mater. Res. 32 113-40

[41] Emmerich H 2003 The Diffuse Interface Approach in Materials Science (Heidelberg: Springer)

[42] Lu Y X, Kreller C and Adler S B 2009 J. Electrochem. Soc. 156 B513-25

[43] Voigt A and Lowengrub J 2009 private communication

[44] Osher S and Fedkiw R 2003 Level Set Methods and Dynamic Implicit Surfaces (New York: Springer) pp 66-7 
[45] Gurtin M E, Polignone D and Vinals J 1996 Math. Models Methods Appl. Sci. 6 815-31

[46] Jacqmin D 1999 J. Comput. Phys. 155 96-127

[47] Kim J 2005 J. Comput. Phys. 204 784-804

[48] Zhou B and Powell A C 2006 J. Membr. Sci. 268 150-64

[49] Villanueva W, Gronhagen K, Amberg G and Agren J 2008 Phys. Rev. E 77056313

[50] Villanueva W, Boettinger W J, Warren J A and Amberg G 2009 Acta Mater. 57 6022-36

[51] Darken L S 1948 Tran. Am. Inst. Min. Metall. Eng. 175 184-201

[52] Bardeen J and Herring C 1951 Atom Movements-A Seminar on Atom Movements Held During the 32nd Natl Metal Congress and Exposition (Chicago, IL, 21-27 October 1950) (Cleveland, OH: American Society for Metals) p 87-111

[53] Strandlund H and Larsson H 2004 Acta Mater. 52 4695-703

[54] Larsson H, Strandlund H and Hillert M 2006 Acta Mater. 54 945-51

[55] Strandlund H and Larsson H 2006 Metall. Mater. Trans. A 37 1785-9

[56] Yu H-C, Yeon D-H, Van der Ven A and Thornton K 2007 Acta Mater. 55 6690-704

[57] Svoboda J, Fischer F D and Gamsjager E 2008 Acta Mater. 56 351-7

[58] Kirkendall E, Thomassen L and Uethegrove C 1939 Tran. Am. Inst. Min. Metall. Eng. 133 186-203

[59] Kirkendall E O 1942 Tran. Amer. Inst. Min. Met. Eng. 147 104-9

[60] Smigelskas A D and Kirkendall E O 1947 Tran. Am. Inst. Min. Metall. Eng. 171 130-42

[61] Stephenson G B 1988 Acta Metall. 36 2663-83

[62] Boettinger W J, McFadden G B, Coriell S R and Warren J A 2005 Acta Mater. 53 1995-2008

[63] Dantzig J A, Boettinger W J, Warren J A, McFadden G B, Coriell S R and Sekerka R F 2006 Metall. Mater. Trans. A 37 2701-14

[64] Boettinger W J, Guyer J E, Campell C E and McFadden G B 2007 Proc. R. Soc. A-Math. Phy. 463 3347-73

[65] Boettinger W J and McFadden G B 2010 J. Phase Equilib. Diff. 31 6-14

[66] Yu H-C 2009 Rigorous model and simulations of the Kirkendall effect diffusion in substitutional bindary alloys PhD Thesis University of Michigan

[67] Kim D and Lu W 2006 Comput. Mater. Sci. 38 418-25

[68] Kim D and Lu W 2006 J. Mech. Phys. Solids. 54 2554-68

[69] Moleko L K, Allnatt A R and Allnatt E L 1989 Phil. Mag. A 59 141-60

[70] Manning J R 1971 Phys. Rev. B 4 1111-25

[71] Belova I V and Murch G E 2000 Phil. Mag. A 80 599-607

[72] Van der Ven A, Yu H-C, Ceder G and Thornton K 2010 Prog. Mater. Sci. 55 61-105

[73] Wang S R, Katsuki M, Hashimoto T and Dokiya M 2003 J. Electrochem. Soc. 150 A952-8

[74] Sorensen B F and Horsewell A 2001 J. Am. Ceram. Soc. 84 2051-9

[75] Wang Y L, Duncan K, Wachsman E D and Ebrahimi F 2007 Solid State Ion. 178 53-8

[76] Serincan M F, Pasaogullari U and Sammes N M 2010 J. Power Sources 195 4905-14

[77] Steinbach I 2009 Modeling Simul. Mater. Sci. Eng. 17073001

[78] Du Q, Liu C and Wang X 2004 J. Comput. Phys. 198 450-68

[79] Chen H-Y, Yu H-C and Thornton K 2011 J. Power Sources 196 1333-7

[80] Yu H-C, Van der Ven A and Thornton K 2012 Metal. Mater. Trans. A 43A 3481-500

[81] Langer S A, Fuller E and Carter W C 2001 Comput. Sci. Eng. 3 15-23

[82] Thornton K and Poulsen H F 2008 MRS Bull. 33 587-95

[83] Spanos G, Rowenhorst D J, Lewis A C and Geltmacher A B 2008 MRS Bull. 33 597-602

[84] Park C L, Voorhees P W and Thornton K 2010 private communication

[85] Sussman M, Smereka P and Osher S 1994 J. Comput. Phys. 114 146-59

[86] Osher S and Fedkiw R 2003 Level Set Methods and Dynamics Implicit Surfaces (New York: Springer) p 54

[87] Van de Velde E F 1994 Concurrent Scientific Computing 1st edn (New York: Springer) p 202

[88] Hofhaus J and Van de Velde E F 1996 SIAM J. Sci. Comput. 17 454-78 